\title{
HAWKING RADIATION AND BLACK HOLE THERMODYNAMICS *
}

\author{
Don N. Page ${ }^{\dagger}$ \\ Institute for Theoretical Physics \\ Department of Physics, University of Alberta \\ Edmonton, Alberta, Canada T6G 2J1
}

(2004 September 3; additional section added 2004 December $311^{\ddagger}$ )

\begin{abstract}
An inexhaustive review of Hawking radiation and black hole thermodynamics is given, focusing especially upon some of the historical aspects as seen from the biased viewpoint of a minor player in the field on and off for the past thirty years.
\end{abstract}

*Alberta-Thy-18-04, hep-th/0409024 , review article solicited for a celebratory Focus Issue on Relativity, "Spacetime 100 Years Later," to be published in New Journal of Physics.

†Internet address: don@phys.ualberta.ca

$\ddagger$ On this date the author reached an age which is an integer divisor of the number of references. 


\section{Historical Background}

Black holes are perhaps the most perfectly thermal objects in the universe, and yet their thermal properties are not fully understood. They are described very accurately by a small number of macroscopic parameters (e.g., mass, angular momentum, and charge), but the microscopic degrees of freedom that lead to their thermal behavior have not yet been adequately identified.

Strong hints of the thermal properties of black holes came from the behavior of their macroscopic properties that were formalized in the (classical) four laws of black hole mechanics [1], which have analogues in the corresponding four laws of thermodynamics:

The zeroth law of black hole mechanics is that the surface gravity $\kappa$ of a stationary black hole is constant over its event horizon [2, 1]. This is analogous to the zeroth law of thermodynamics, that the temperature $T$ is constant for a system in thermal equilibrium.

The first law of black hole mechanics expresses the conservation of energy by relating the change in the black hole mass $M$ to the changes in its area $A$, angular momentum $J$, and electric charge $Q$ in the following way:

$$
\delta M=\frac{1}{8 \pi} \kappa \delta A+\Omega \delta J+\Phi \delta Q
$$

where an extended form of the zeroth law implies that not only the surface gravity $\kappa$, but also the angular velocity $\Omega$ and the electrostatic potential $\Phi$ are constant over the event horizon of any stationary black hole. This first law is essentially the same as the first law of thermodynamics.

The second law of black hole mechanics is Hawking's area theorem [3], that the area $A$ of a black hole horizon cannot decrease. This is obviously analogous to the second law of thermodynamics, that the entropy $S$ of a closed system cannot decrease.

The third law of black hole mechanics is that the surface gravity $\kappa$ cannot be reduced to zero by any finite sequence of operations [4]. This is analogous to the weaker (Nernst) form of the third law of thermodynamics, that the temperature $T$ of a system cannot be reduced to absolute zero in a finite number of operations. However, the classical third law of black hole mechanics is not analogous to the stronger (Planck) form of the third law of thermodynamics, that the entropy of a system goes to zero when the temperature goes to zero. 
Thus the four laws of black hole mechanics are analogous to the four laws of thermodynamics if one makes an analogy between temperature $T$ and some multiple of the black hole surface gravity $\kappa$, and between entropy $S$ and some inversely corresponding multiple of the black hole area $A$. That is, one might say that $T=\epsilon \kappa$ and $S=\eta A$, with $8 \pi \epsilon \eta=1$, so that the $\kappa \delta A /(8 \pi)$ term in the first law of black hole mechanics becomes the heat transfer term $T \delta S$ in the first law of thermodynamics.

Even before the formulation of the four laws of black hole mechanics, Bekenstein [5, 6, 7, 8, proposed that a black hole has an entropy $S$ that is some finite multiple $\eta$ of its area $A$. He was not able to determine the exact value of $\eta$, but he gave heuristic arguments for conjecturing that it was $(\ln 2) /(8 \pi)$ (in Planck units, $\hbar=$ $c=G=k=4 \pi \epsilon_{0}=1$, which I shall use throughout).

However, for the first law of black hole mechanics to be equivalent to the first law of thermodynamics, this would logically imply that the black hole would have to have a temperature $T$ that is a corresponding nonzero multiple of the surface gravity $\kappa$. E.g., if $\eta=(\ln 2) /(8 \pi)$ as Bekenstein proposed, then one would get $\epsilon=1 /(\ln 2)$, so that $T=\kappa /(\ln 2)$. But since it was thought then that black holes can only absorb and never emit, it seemed that black holes really would have zero temperature, or $\epsilon=0$, which would make Bekenstein's proposal inconsistent with any finite $\eta$ [1].

Nevertheless, by a quite independent line of reasoning that was not directly motivated by Bekenstein's proposal that he had rejected [1], Hawking made the remarkable discovery that black holes are not completely black but instead emit radiation [9, 10]. Once he found that the radiation had a thermal spectrum, he realized that it did make Bekenstein's idea consistent, of a finite black hole entropy proportional to area, though not Bekenstein's conjectured value for $\eta$. In fact, Hawking found that the black hole temperature was $T=\kappa /(2 \pi)$, so $\epsilon=1 /(2 \pi)$ and hence $\eta=1 / 4$. This gives the famous Bekenstein-Hawking formula for the entropy of a black hole:

$$
S_{\mathrm{bh}}=S_{\mathrm{BH}} \equiv \frac{1}{4} A .
$$

Here the subscript bh stands for "black hole," and the subscript BH stands for "Bekenstein-Hawking."

A precursor of Hawking's discovery of emission by black holes were the calculations by Parker [11, 12, 13, 14] and Fulling [15, 16] of particle creation by expanding universes, which developed the concepts of Bogoliubov transformations [17] in timedependent geometries that were later used by Hawking. However, to the best of my 
knowledge, it was a surprise to everyone, including Hawking, that the emission from a black hole persisted even when the black hole became effectively static.

Since I did my Ph.D. thesis [18] on "Accretion into and Emission from Black Holes" (but missed the opportunity to use the more catchy title, "The Ins and Outs of Black Holes") and had many discussions about it with Hawking during the 1974-75 year that he spent at Caltech, it may be of interest to quote part of an introductory section from that thesis on what I then saw as the historical background of black hole emission (and now add a few more comments within square brackets):

The first prediction of emission by a black hole was made by Zel'dovich [19, 20]. He pointed out on heuristic grounds that a rotating black hole should amplify certain waves and there should be an analogous quantum effect of spontaneous radiation of energy and angular momentum. Later Misner 21] and Starobinsky 22] confirmed the amplification by a Kerr hole of scalar waves in the "superradiant regime" (where the angular velocity of the wavefronts is lower than that of the waves), and Bekenstein [23] showed that amplification should occur for all kinds of waves with positive energy density. However, the quantum effect predicted by Zel'dovich was not universally known, and in fact Larry Ford at Princeton University and I independently rediscovered it.

The argument for this spontaneous radiation was that in a quantum analysis the amplification of waves is stimulated emission of quanta, so that even in the absence of incoming quanta one should get spontaneous emission. By using the relation between the Einstein coefficients for spontaneous and stimulated emission, one can calculate the spontaneous rate from the amplification factor, as Starobinsky [22] noted, at least when the spontaneous emission probability is much less than unity.

A problem arose for neutrinos in that Unruh [24] showed that their waves are never amplified. This result violated Bekenstein's conclusion and seemed to be a breakdown in the Hawking [3] area theorem. The reason for the violation was traced to a negative local energy density of the classical neutrino waves at the horizon. However, Feynman suggested (unpublished) that the lack of amplification might be due to the Pauli exclusion principle, so that incident neutrinos suppress spontaneous emission which otherwise occurs. The amplification factor would 
then be less than unity, since the calculation of an unquantized neutrino wave cannot directly show the spontaneous emission but only how the emission changes as the incident flux is varied.

[I had met up with Feynman at lunch at Caltech that day in early 1973 to ask him about my idea of spontaneous emission from rotating black holes, just hours before I became somewhat crestfallen to find in [19] that Zel'dovich had already pointed out this effect. Feynman volunteered to come over, after stopping off to watch some belly dancers on campus that day, to the offices of Kip Thorne's graduate students Bill Press, Saul Teukolsky, and me to discuss, and eventually agree with, my idea that at that time I still thought was original. When presented with the problem with the lack of amplification for the classical neutrino waves, Feynman began drawing diagrams on the blackboard, while noting, "I'm supposed to be good at these diagrams."]

One might be surprised to find such a difference between integral and half-integral spins showing up in the behavior of their unquantized waves, but this is merely an illustration of the connection between spin and statistics. Pauli 25] has shown that half-integral spins must be assigned anticommutation relations in order to get a positive energy density, which is precisely what the unquantized neutrino waves violate in not showing superradiance.

Indeed, this same behavior occurs in the Klein paradox. A scalar wave incident on an electrostatic potential step higher than the kinetic energy plus twice the mass gives a reflected current greater than the incident current. On the other hand, a Dirac wave on such a step gives less reflected current. (This is the result if one makes the causality requirement of the transmitted waves' having a group velocity away from the step, rather than having the momentum vector away from the step as in Bjorken and Drell [26].) Nikishov [27] uses field theory to calculate the pair production by a potential step of general shape with no particles incident. His results show that the number of expected particles emitted in a given Klein-paradox state is

$$
\langle N\rangle= \pm(A-1)
$$

where $A$ is the amplification factor for the reflected wave of the unquan- 
tized Klein-Gordon $(+)$ or Dirac $(-)$ equation. This formula applies even if the emission probabilities are not small, so that $\langle N\rangle$ includes the possibility of emitting more than one particle (if a boson) in the same state.

Unruh 28 made a formal calculation of second quantization of scalar and neutrino fields in the complete Kerr metric and found essentially the same results as Eq. (3) if he chose the initial vacuum state to correspond to no particles coming out of the past horizon. Ford [29] quantized the massive scalar field in a somewhat different way with similar results. However, Unruh noted that the actual situation might be different, with no past horizon but the black hole formed by collapse. Nevertheless, neither he nor any of the discoverers of the spontaneous emission attempted to calculate that situation.

Meanwhile (summer 1973), Stephen Hawking at Cambridge University heard of this work through Douglas Eardley [who as a postdoc at Caltech had learned of it from discussions with Press, Teukolsky, and me] and so while in Moscow discussed it with Zel'dovich and Starobinsky. Believing in the reality of the spontaneous emission but wishing to put its derivation on a firmer footing, Hawking dared to attempt the difficult calculation of field theory during the collapse and formation of a black hole. Separating out the essential elements, Hawking found how to calculate the particle emission at late times, after the collapse had settled down to form a stationary black hole. At first Hawking got an infinite number of particles emitted, but then he discovered that the infinity corresponded to emission at a steady rate. However, the emission was not only in the superradiant states or modes but in all modes that could come from the black hole!

Hawking initially did not believe this result (a consolation to those of us who doubted it also when we first heard it). Thinking that the emission might be an artifact of the spherical symmetry he had assumed, Hawking considered nonspherical collapse and got the same emission. Then he tried putting in a cutoff on the frequencies of the modes in the initial state before the collapse, but that eliminated all the emission, including the spontaneous emission in the superradiant modes that Hawking was certain existed. Perhaps most convincing to Hawking was 
the fact that the emission rate was just that of a thermal body with the same absorption probabilities as the black hole and with a temperature (in geometrical units) equal to the surface gravity of the hole divided by $2 \pi$. This result held for fields of any spin and seemed to confirm some thermodynamic ideas of Bekenstein [7]. However, before the emission process was discovered, Bardeen, Carter, and Hawking [1] had argued against Bekenstein's suggestion of a black-hole temperature proportional to surface gravity. Thus Bekenstein's ideas were originally not a motivation for Hawking's calculation.

As word of his calculation began to spread, Hawking published a simplified version of it in Nature [9]. However, even at this stage Hawking was not certain of the result and so expressed the title as a question, "Black hole explosions?" He noted that the calculation ignored the change in the metric due to the particles created and to quantum fluctuations. One objection raised by several people was that the calculation seemed to give a very high energy flux just outside the horizon, which might prevent the black hole from forming at all. Hawking later answered this and other problems by a more detailed version of the calculation [10, which showed that an infalling observer would not see many particles near the horizon. However, it might be noted that there is still some controversy about the existence of particles there. The back reaction of the particles created would, in Hawking's view, simply be to reduce the mass of the hole by the amount of the energy radiated away.

Presumably quantum fluctuations of the metric itself can give rise to the emission of gravitons in addition to the emission of other particles calculated as if the geometry were fixed. By considering linearized fluctuations in the metric about a given background, the emission of gravitons can be handled in the same manner as the emission of any other particles, though one might argue that graviton emission depends more fundamentally upon the assumed fluctuations in the metric. Therefore, any observed consequences of graviton emission can be viewed as testing whether gravity is quantized.

Hawking has argued (unpublished) that quantum mechanics allows small deviations of the action from the extremum value that gives the classical field equations for matter and geometry. Thus the classical 
equations can be violated in a small region near a black hole, giving rise to the emission of matter or gravitational waves, but the equations cannot be violated significantly on a very large surface surrounding the hole. Therefore, quantities determined by surface fluxes at infinity do remain conserved: energy, momentum, angular momentum, and charge. This is the basis for arguing that the emission carries away the quantities of the hole which otherwise would be constant. Note that baryon and lepton numbers are not observed to be connected with long-range fields, so they presumably cannot be determined by surface fluxes at infinity and thus would not be conserved globally by the black-hole emission process.

The thermal emission first calculated by Hawking has been verified by several subsequent calculations. Boulware [30] and Davies [31] have calculated the emission from a collapsing shell. Gerlach 32 has interpreted the emission as parametric amplification of the zero-point oscillations of the field inside the collapsing object. DeWitt 33] has given detailed derivations of both the spontaneous emission process in the complete Kerr metric (with no particles coming out of the past horizon) and of the thermal emission from a black hole formed by collapse. Unruh 34 has found that his derivation in the complete Kerr metric will give not only the spontaneous but also the thermal emission if the boundary condition at the past horizon is changed from no particles seen by an observer at fixed radius just outside the horizon to no particles seen by an observer freely falling along the horizon. Wald [35], Parker [36] and Hawking [37] have calculated the density matrix of the emitted particles and find that it, as well as the expected number in each mode, is precisely thermal. [For a time Hawking thought that particles escaped only in pairs, which led me to come back from a spring camping trip in the snow in the Sierras in 1975 thinking I had figured out how to violate the second law of thermodynamics. Never have I made that error again.] Bekenstein 38, has given an information-theory argument of why this should be so. Hartle and Hawking 39] have done a path-integral calculation of the probability for a particle to propagate out of a black hole from the future singularity and show that this method also leads to the same thermal spectrum. In summary, the thermal emission from 
a black hole has been derived in a variety of ways by several people, so its prediction seems to be a clear consequence of our present theories of quantum mechanics and general relativity.

That of course was my own personal view as I was finishing my Ph.D. in 1976, heavily influenced by discussions with Hawking and with a few others, but without in any way being a claim to a completely balanced and broad view of what others might have been thinking at the time. However, I thought it might be of at least some historical interest to present here this biased viewpoint of the important historical development of black hole emission. For other viewpoints, see [40, 41, 42].

\section{Hawking Emission Formulae}

For the Kerr-Newman metrics [43, 44], which are the unique asymptotically flat stationary black holes in Einstein-Maxwell theory [45, 46, 47, 48, 49, one can get explicit expressions for the area $A$, surface gravity $\kappa$, angular velocity $\Omega$, and electrostatic potential $\Phi$ of the black hole horizon in terms of the macroscopic conserved quantities of the mass $M$, angular momentum $J \equiv M a \equiv M^{2} a_{*}$, and charge $Q \equiv M Q_{*}$ of the hole [50, using the value $r_{+}$of the radial coordinate $r$ at the event horizon as an auxiliary parameter:

$$
\begin{aligned}
r_{+} & =M+\left(M^{2}-a^{2}-Q^{2}\right)^{1 / 2}=M\left[1+\left(1-a_{*}^{2}-Q_{*}^{2}\right)^{1 / 2}\right] \\
A & =4 \pi\left(r_{+}^{2}+a^{2}\right)=4 \pi M^{2}\left[2-Q_{*}^{2}+2\left(1-a_{*}^{2}-Q_{*}^{2}\right)^{1 / 2}\right] \\
\kappa & =\frac{4 \pi\left(r_{+}-M\right)}{A}=\frac{1}{2} M^{-1}\left[1+\left(1-\frac{1}{2} Q_{*}^{2}\right)\left(1-a_{*}^{2}-Q_{*}^{2}\right)^{-1 / 2}\right]^{-1} \\
\Omega & =\frac{4 \pi a}{A}=a_{*} M^{-1}\left[2-Q_{*}^{2}+2\left(1-a_{*}^{2}-Q_{*}^{2}\right)^{1 / 2}\right]^{-1} \\
\Phi & =\frac{4 \pi Q r_{+}}{A}=Q_{*} \frac{1+\left(1-a_{*}^{2}-Q_{*}^{2}\right)^{1 / 2}}{2-Q_{*}^{2}+2\left(1-a_{*}^{2}-Q_{*}^{2}\right)^{1 / 2}}
\end{aligned}
$$

Here $a_{*}=a / M=J / M^{2}$ and $Q_{*}=Q / M$ are the dimensionless angular momentum and charge parameters in geometrical units $\left(G=c=k=4 \pi \epsilon_{0}=1\right.$ but for this without setting $\hbar=1$, so that mass, time, length, and charge all have the same units, and angular momentum has units of mass or length squared; e.g., the angular momentum of the sun is $J_{\odot} \equiv a_{* \odot} M_{\odot}^{2}=(0.2158 \pm 0.0017) M_{\odot}^{2}=47.05 \pm 0.37$ hectares $=$ $116 \pm 1$ acres [51, 52]. However, we shall return to Planck units for the rest of this 
paper, so that every quantity is dimensionless.) For a nonrotating uncharged stationary black hole (described by the Schwarzschild metric), $a_{*}=Q_{*}=0$, so $r_{+}=2 M$, $A=16 \pi M^{2}, \kappa=M / r_{+}^{2}=1 /(4 M), \Omega=0$, and $\Phi=0$.

Then Hawking's black hole emission calculation [9, 10] for free fields gives the expected number of particles of the $j$ th species with charge $q_{j}$ emitted in a wave mode labeled by frequency or energy $\omega$, spheroidal harmonic $l$, axial quantum number or angular momentum $m$, and polarization or helicity $p$ as

$$
N_{j \omega l m p}=\Gamma_{j \omega l m p}\left\{\exp \left[2 \pi \kappa^{-1}\left(\omega-m \Omega-q_{j} \Phi\right)\right] \mp 1\right\}^{-1} .
$$

Here the upper sign (minus above) is for bosons, and the lower sign (plus above) is for fermions, and $\Gamma_{j \omega l m p}$ is the absorption probability for an incoming wave of the mode being considered.

More accurately, $\Gamma_{j \omega l m p}$ is the negative of the fractional energy gain in a scattered classical wave with only inward group velocity at the black hole horizon. $\Gamma_{j \omega l m p}$ is positive for all fermionic wave modes and for bosonic wave modes with $\omega-m \Omega-$ $q_{j} \Phi>0$, which are at least partially absorbed by the hole, but it is negative for bosonic superradiant modes with $\omega-m \Omega-q_{j} \Phi<0$, which are amplified by the hole. Thus $0 \leq \Gamma_{j \omega l m p} \leq 1$ for fermionic modes, but one just has $\Gamma_{j \omega l m p} \leq 1$ for bosonic modes, with $\Gamma_{j \omega l m p}$ allowed to be negative for them.

Nevertheless, $N_{j \omega l m p}$ is never negative, because the thermal Planck factor is also negative for bosonic superradiant modes. $N_{j \omega l m p}$ also never diverges, even though the Planck factor for bosons diverges as $\omega-m \Omega-q_{j} \Phi$ is taken to zero, since then $\Gamma_{j \omega l m p}$ also goes to zero linearly with $\omega-m \Omega-q_{j} \Phi$ and so keeps $N_{j \omega l m p}$ finite. Then one can combine $\Gamma_{j \omega l m p} \leq 1$ with Eq. (15) to get the double inequality

$$
\mp N_{j \omega l m p} \leq \Gamma_{j \omega l m p} \leq 1
$$

In the approximation of a stationary geometry with no back reaction, the density matrix of the Hawking radiation is (for free fields) the uncorrelated tensor product of thermal density matrices for each of the modes with definite frequency, angular momentum, and charge. The thermal density matrices for each mode are diagonal in the number basis, with the probability of $n$ particles in the mode being

$$
P_{n}=N^{n}(1 \pm N)^{-n \mp 1}
$$

where for brevity I have here dropped the mode-labeling subscripts on the expected number $N_{j \omega l m p}$ of particles in the mode. Here $n$ can be any nonnegative integer for bosons (upper sign) but is restricted to be 0 or 1 for fermions (lower sign). 
The von Neumann entropy for the thermal density matrix of each mode is

$$
\delta S_{\mathrm{rad}}=-\sum_{n} P_{n} \ln P_{n}=(N \pm 1) \ln (1 \pm N)-N \ln N .
$$

Since the expected loss of energy, angular momentum, and charge of the hole from emitting $N$ particles in the mode are $N \omega, N m$, and $N q_{j}$ respectively, the expected change in the black hole entropy from that emission mode is

$$
\delta S_{\mathrm{bh}}=-N\left[2 \pi \kappa^{-1}\left(\omega-m \Omega-q_{j} \Phi\right)\right]=-N \ln \left(\frac{\Gamma \pm N}{N}\right),
$$

where now I have omitted the mode-labeling subscripts not only on $N_{j \omega l m p}$, but also on $\Gamma_{j \omega l m p}$.

Then the total expected change in the entropy of the world from the emission of the mode in question is

$$
\delta S=\delta S_{\mathrm{rad}}+\delta S_{\mathrm{bh}}= \pm \ln (1 \pm N)+N \ln \left(1+\frac{1-\Gamma}{\Gamma \pm N}\right) \geq \pm \ln (1 \pm N) \geq 0,
$$

with the extreme right inequality being saturated only if there is no emission, $N=0$.

Thus the Hawking emission from a black hole into empty space obeys the second law of thermodynamics, and it actually produces entropy from all modes with nonzero emission. This is as one would expect, since the emission from a black hole with $T_{\mathrm{bh}}>0$ into empty space with $T=0$ is an out-of-equilibrium process.

It is important to note that since the expected number of particles $N \equiv N_{j \omega l m p}$ depends not only on the Planck factor but also on $\Gamma_{j \omega l m p}$, the effective temperature $T_{j \omega l m p}$ varies from mode to mode. The effective temperature may be defined by the Boltzmann factor

$$
\frac{P_{1}}{P_{0}} \equiv e^{-\omega / T_{j \omega l m p}}
$$

Since $P_{1} / P_{0}=N /(1 \pm N)$, one gets

$$
T_{j \omega l m p}=\omega / \ln \left(\frac{1 \pm N_{j \omega l m p}}{N_{j \omega l m p}}\right) .
$$

When $m \Omega+q_{j} \Phi=0$ (e.g., for the Schwarzschild metric, but also for s-waves of neutral particles in any Kerr-Newman geometry), so that the Planck factor becomes simply $1 /\left(e^{2 \pi \omega / \kappa} \mp 1\right)$, and when $\Gamma_{j \omega l m p}=1$, so that the classical incoming wave is totally absorbed by the black hole, then $T_{j \omega l m p}=T_{\mathrm{bh}}=\kappa /(2 \pi)$, the Hawking temperature of the hole. But otherwise, the effective temperature $T_{j \omega l m p}$ for the mode generically depends on the mode. 
For example, when $m \Omega+q_{j} \Phi=0$, then generically $\Gamma_{j \omega l m p}<1$ and $T_{j \omega l m p}<T_{\mathrm{bh}}$. For modes that have sufficiently large angular momentum in comparison with their energy, so that they mostly miss the black hole and have negligible absorption probability $\Gamma_{j \omega l m p}$, the effective temperature is much less than the actual black hole temperature.

On the other hand, for nearly extreme black holes, $1-a_{*}^{2}-Q_{*}^{2} \ll 1$, which have low temperatures, $T_{\mathrm{bh}} \ll 1 /(8 \pi M)$, the latter being the Schwarzschild value, and for modes with $\omega-m \Omega-q_{j} \Phi<0$ (both for bosonic superradiant modes with $\Gamma_{j \omega l m p}<0$ and for these fermionic modes that still have $\Gamma_{j \omega l m p}>0$ ), one can have $T_{j \omega l m p} \gg T_{\mathrm{bh}}$. This is the case in which the temperature of the black hole has a negligible effect, and the Hawking emission formula reduces approximately to Eq. (3) above (where the amplification factor is $A=-\Gamma_{j \omega l m p}$ ) for the spontaneous emission first discovered by Zel'dovich [19, 20].

From the mean number $N_{j \omega l m p}$ and the entropy $S_{j \omega l m p}$ per mode, one can sum and integrate over modes to get the emission rates of energy, angular momentum (the component parallel to the black hole spin axis), charge, and entropy by the black hole:

$$
\begin{gathered}
\frac{d E_{\mathrm{rad}}}{d t}=-\frac{d M}{d t}=\frac{1}{2 \pi} \sum_{j, l, m, p} \int \omega N_{j \omega l m p} d \omega, \\
\frac{d J_{\mathrm{rad}}}{d t}=-\frac{d J}{d t}=\frac{1}{2 \pi} \sum_{j, l, m, p} \int m N_{j \omega l m p} d \omega, \\
\frac{d Q_{\mathrm{rad}}}{d t}=-\frac{d Q}{d t}=\frac{1}{2 \pi} \sum_{j, l, m, p} \int q_{j} N_{j \omega l m p} d \omega, \\
\frac{d S_{\mathrm{rad}}}{d t}=\frac{1}{2 \pi} \sum_{j, l, m, p} \int S_{j \omega l m p} d \omega .
\end{gathered}
$$

Here $M, J$, and $Q$ (without subscripts) denote the black hole's energy, angular momentum, and charge. By the conservation of the total energy, angular momentum, and charge, the black hole loses these quantities at the same rates that the radiation gains them.

This is not so for the total entropy, which generically increases, as noted above. The black hole entropy changes at the rate

$$
\frac{d S_{\mathrm{bh}}}{d t}=\frac{1}{2 \pi} \sum_{j, l, m, p} \int\left[2 \pi \kappa^{-1}\left(\omega-m \Omega-q_{j} \Phi\right)\right] N_{j \omega l m p} d \omega,
$$


and by using Eq. (5), as in the derivation of Eq. (10), one can show that the total entropy $S=S_{\mathrm{bh}}+S_{\mathrm{rad}}$ (black hole plus radiation) changes at the rate

$$
\frac{d S}{d t}=\frac{1}{2 \pi} \sum_{j, l, m, p} \int d \omega\left[ \pm \ln \left(1 \pm N_{j \omega l m p}\right)+N_{j \omega l m p} \ln \left(1+\frac{1-\Gamma_{j \omega l m p}}{\Gamma_{j \omega l m p} \pm N_{j \omega l m p}}\right)\right] \text {. }
$$

For the emission of $n_{s}$ species of two-polarization massless particles of spin $s$ from a Schwarzschild black hole (nonrotating and uncharged) into empty space, numerical calculations [50, 53, 54] gave

$$
\begin{gathered}
\frac{d E_{\mathrm{rad}}}{d t}=-\frac{d M}{d t}=10^{-5} M^{-2}\left(8.1830 n_{1 / 2}+3.3638 n_{1}+0.3836 n_{2}\right), \\
\frac{d S_{\mathrm{rad}}}{d t}=10^{-3} M^{-1}\left(3.3710 n_{1 / 2}+1.2684 n_{1}+0.1300 n_{2}\right), \\
\frac{d S_{\mathrm{bh}}}{d t}=-10^{-3} M^{-1}\left(2.0566 n_{1 / 2}+0.8454 n_{1}+0.0964 n_{2}\right) .
\end{gathered}
$$

Therefore, if a Schwarzschild black hole emitted just massless neutrinos into empty space, the entropy in the radiation would be 1.6391 times as much as the entropy decrease of the black hole; if it emitted just photons, the radiation entropy would be 1.5003 times that by which the hole decreased; if it emitted just gravitons, the external entropy would be 1.3481 times the entropy drawn out of the hole; and if three massless neutrino species were emitted along with photons and gravitons (and negligible other particles), the radiation entropy would be larger by a factor of 1.6187 [54].

\section{The Generalized Second Law}

Even if a black hole is not emitting into empty space, there are strong arguments that the total entropy of the black hole plus its environment cannot decrease. This is the Generalized Second Law (GSL). Bekenstein first conjectured it when he proposed that black holes have finite entropy proportional to their area [5, 6, 7, 8, and he gave various arguments on its behalf, though it would have been violated by immersing a black hole in a heat bath of sufficiently low temperature if the black hole could not emit radiation [1].

Once Hawking found that black holes radiate [9, 10, he showed that the GSL held for a black hole immersed in a heat bath of arbitrary temperature, assuming that the radiation thermalized to the temperature of the heat bath. Zurek and Thorne [55], 
and Thorne, Zurek, and Price [56], gave more general arguments for the GSL without this last assumption. Their arguments were later fleshed out in a mathematical proof of the GSL for any process involving a quasistationary semiclassical black hole [57]. Other proofs of the GSL have also been given [58, 59, 60, 61.

With some exceptions [59, 61], these proofs so far generally have two key assumptions: (1) The black hole is assumed to be quasistationary, changing only slowly during its interaction with an environment. It has been conjectured [56] that the GSL also holds, using the Bekenstein-Hawking $A / 4$ formula for the black hole entropy, even for rapid changes in the black hole, but this has not been rigorously proved. Even to make this conjecture precise would require a precise definition of the entropy of the environment, which is problematic in quantum field theory when one attempts to define the entropy of quantum fields in some partial region of space (e.g., the region outside the black hole) with a sharp boundary [62, 63.

(2) The semiclassical approximation holds, so that the black hole is described by a classical metric which responds only to some average or expectation value of the quantum stress-energy tensor. This allows the black hole entropy to be represented by $A / 4$ of its classical horizon. This approximation also implies that the radiation from the hole is essentially thermal, with negligible correlations between what is emitted early and late in the radiation, so that one may use the von Neumann entropy $S_{\mathrm{rad}}=-\operatorname{tr}(\rho \ln \rho)$ for the entropy of the radiation and yet have it plus $A / 4$ for the black hole to continue to increase (once a suitable way is chosen to regularize the divergence of $-\operatorname{tr}(\rho \ln \rho)$ that one would get from a sharp black hole boundary 62, 63]).

Now if information is really lost down a black hole as Hawking originally proposed [64, and if the Hawking radiation really has negligible correlations between what is emitted early and late, then it might be true that $A / 4-\operatorname{tr}(\rho \ln \rho)$, suitably regularized, would never decrease. But since this information loss proposal has been controversial since near the beginning [65, 66, and since now even Hawking has given it up [67, it might well be that information is not lost forever down a black hole but instead comes back out with the radiation. If so, for a black hole formed from matter in nearly a pure state, the total radiation from the hole (after it evaporates completely, as I assume it will) will also be in nearly a pure state. In this case, when the black hole originally formed, the total entropy in the GSL would be somewhat greater than $A / 4$ and hence large. However, after the evaporation, there would be no black hole entropy, and the radiation, in nearly a pure state, would have 
very little entropy. Thus the total GSL entropy would have gone down enormously.

Of course, this same problem could arise in the second law for any other composite system if the entropy were taken to be the sum of the von Neumann entropies for each of the subsystems. For example, if one had a lump of coal in nearly a pure state with incoming radiation also in nearly a pure state, the radiation could heat up the coal, which would then radiate nearly thermally. When the hot coal had only partially cooled, there would be sufficient correlations between what the coal had radiated and its own internal state that the von Neumann entropy of both would be large. Thus, by this coarse-grained procedure of calculating the entropy of the coal plus the radiation (by ignoring the entanglement or quantum correlations between these two subsystems and just adding the von Neumann entropies of the density matrices of each that are obtained by tracing over the rest of the total system), one would get a high entropy at intermediate times. However, at late times, the coal would cool back down to nearly its ground state of low entropy, and the radiation would similarly be nearly a pure state (with a large amount of subtle correlations between its different parts), so the total von Neumann entropy of these two parts would have decreased back down to a small value again.

This phenomenon illustrates the problem that nontrivial versions of the second law usually require coarse graining, but then the result depends on the coarse graining and may not always have the desired property. If one uses the von Neumann entropy of an entire closed system with no coarse graining, then if this system evolved unitarily (e.g., with no loss of information), then the von Neumann entropy is simply a constant, and the second law becomes trivial. Dividing a total system up into subsystems, calculating the density matrix and von Neumann entropy of each part by tracing over the rest of the system, and then adding up the resulting entropies of each part usually does give a nontrivial entropy by this coarse graining that ignores the quantum correlations or entanglements between the subsystems. This nontrivial entropy can indeed increase if correlations between the subsystems grow, so that more of the quantum information about the total system goes into the correlations that are ignored in this particular coarse-grained method of calculating the entropy.

Typically in our universe spatially separated subsystems have less than maximal correlations between them, and typically interactions between these subsystems cause the correlations to grow with time. In this way the coarse graining that ignores these correlations gives an increasing entropy and expresses the second law of ther- 
modynamics for our universe. However, there can be exceptions, such as the coal that cools off so that it no longer has the energy to remain significantly correlated with the radiation it emitted. (In this example one could save the second law by dividing the radiation itself up into subsystems whose correlations do not decrease with time, but this example illustrates that the validity of this formulation of the second law depends on the choice of coarse graining and may be violated for certain choices.)

Since there are these problems with ordinary systems in giving a precise nontrivial definition of entropy that always obeys the second law of thermodynamics, one should not be too surprised that there may also be problems with formulations of the second law for systems containing black holes. Therefore, it is probably unrealistic to expect that there can be a rigorous proof of the second law (or of a GSL) for black hole systems in all generality.

Nevertheless, we would expect that if we have a sufficient amount of coarse graining (such as coarse graining the radiation or other black hole environment into sufficiently many parts and ignoring their correlations, as well as ignoring the correlations between the radiation and the black hole), the GSL should almost always be valid. This people have tested with a wide variety of gedanken experiments.

Before Hawking had discovered that black holes radiate, Bekenstein [7, 8] realized that his GSL might be violated if an entropy-carrying object could be lowered sufficiently near a black hole (so that nearly all of its energy could be extracted first) and then dropped in with its energy so low that the increase of the black hole entropy would not balance the loss of the entropy of the object. To avoid this violation of the GSL, Bekenstein proposed that there was a limit on how close to the black hole an object with fixed entropy and fixed local energy could be lowered. This led Bekenstein to conjecture [68 that the entropy $S$ of a system of energy $E$ and linear size $R$ was limited by the formula

$$
S \leq S_{B}(E, R) \equiv 2 \pi E R
$$

This was a very interesting proposal in its own right, but it developed that there are a lot of problems with it [69, 70, 71, 72, 73, 74, 75, 76, 77, 78, 79]. Perhaps the main difficulty is how to give precise definitions for the system and for its $S, E$, and $R$ [78. For various choices of those definitions, one could easily come up with counter-examples to the conjecture.

For example, if the system is arbitrary quantum fields in some bounded region 
of space, and if zero-point energies are not counted in $E$ (or else one could violate the bound by negative Casimir energies [69]), then by making the number of fields $N$ sufficiently large, $S$ grows as $\ln N$ for fixed $E$ and $R$, allowing the bound to be violated [70, 71]. Or, if $E$ is the expectation value of the energy (over the groundstate value), then by taking a density matrix formed from the ground state and a tiny mixture of an excited state with small probability $p, E$ goes linearly with $p$, but $S$ goes as $p \ln (1 / p)$, so $S / E \sim \ln (1 / p)$, which diverges as $p \rightarrow 0$ and is not bounded by $2 \pi R[72,76,77,78]$. Also, if the bounded region of space is sufficiently nonspherical, one can get violations with large $S \gg 1 \gg S_{B} \equiv 2 \pi E R$ even with small $N$ [76, 77].

Bekenstein has given rebuttals [80, 81, 82, 83, 84] that the system should be a complete system with positive energy, so that presumably $S_{B}$ is not to be allowed to become arbitrarily small. In that case, in which $S_{B}$ is larger than some number that depends on the number of fields $N$ etc., it does seem plausible that the actual entropy $S$ may be bounded by $S_{B}$. For example, for thermal radiation in 4-dimensional spacetime, for $S_{B} \gg 1$ one gets $S \sim S_{B}^{3 / 4}$ (with a coefficient depending on the number of fields etc.), which is certainly bounded by $S_{B}$ for sufficiently large $S_{B}$. However, I would be skeptical that there is any reasonable definition of $S, E$, and $R$ that allows $S_{B} \equiv 2 \pi E R$ to be arbitrarily small and yet maintains the bound $S \leq S_{B}$

Furthermore, if one follows Bekenstein's philosophy of considering only complete systems of presumably bounded energy and momentum, it is hard to see how to give a precise definition of the size $R$ that would be finite if it encompassed the entire system, because of the position-momentum uncertainty principle. But if one takes a definition of $R$ that makes it infinite, then $S_{B}$ becomes infinite, and Bekenstein's conjectured bound becomes trivial.

I have proposed a definition of systems with finite $R$ that are "vacuum outside $R$ " 78, but then when $S_{B}$ is made arbitrarily small and one uses the von Neumann definition for $S, S>S_{B}$, so Bekenstein's conjectured bound is violated. One might want to use instead a microcanonical definition of entropy, but that is difficult to do for a finite-size complete system (even if just "vacuum outside $R$ "), since then the system cannot be composed of any finite number of energy eigenstates and hence could not be ascribed any finite microcanonical entropy.

For further work on Bekenstein's conjectured bound and its relation to Raphael Bousso's covariant entropy bound [85, 86, 87, 88, 89], see [90, 91, 92, 93, 94]. 
However, here the question is to what extent Bekenstein's proposed bound is related to the Generalized Second Law. Unruh and Wald and others [71, 73, 74, 75] have argued that what saves the GSL is not Bekenstein's proposed bound, but the buoyancy or flotations effect of Hawking radiation, which prevents one from lowering an object close enough to the black hole that one can extract enough energy from it to give a violation of the GSL when the object falls in.

(As an historical aside, after the Unruh-Wald buoyancy mechanism was published in 1982, I recalled privately proposing to Wald in early 1976 this mechanism for saving the GSL, but in 1976 it was not believed that the Hawking radiation near the black hole horizon would really be observable or have any significant buoyancy effect, so my proposal seemed untenable and was dropped from consideration at that time. Wald more recently told me he did not remember my suggestion when he and Unruh independently rediscovered this mechanism after realizing that the Unruh acceleration radiation 95] would make the buoyancy effect real. I have no reason at all to doubt his honesty about this, especially since I myself did not remember and publish my own abandoned suggestion even when it became apparent that there would be a real buoyancy effect from the Unruh acceleration radiation. Perhaps the moral of this incident is that even if there is an apparently strong objection to your otherwise good idea, don't dismiss it too completely from your memory.)

Bekenstein [80, 81, 82] has disputed the claim that the buoyancy effect saves the GSL even without his conjectured entropy bound. However, it does seem to be the case that there are several "proofs" of the GSL that do not obviously require assuming Bekenstein's proposed bound, so it seems that surely it is unnecessary (though this argumentation does not rule out the possibility that some form of Bekenstein's proposed bound might follow from some of the same assumptions that implicitly go into the GSL, so that it really is necessary, perhaps somewhat analogous to the way that $2+2=4$ is logically necessary for Einstein's equations to follow from the Einstein-Hilbert action, even though one may not need explicitly to invoke $2+2=4$ in deriving Einstein's equations).

\section{Microscopic Description of Black Hole Entropy}

Even if it turns out that the Generalized Second Law is generally valid under suitable circumstances, there is still the question of what the entropy of a black hole represents. For ordinary thermodynamic systems, the entropy is in some sense roughly 
(the logarithm of) a count of the number of states accessible to the system. That is, if a system has equal probabilities to be in any of $N$ states (and no probability to be in any other states), then its entropy is $\ln N$. Of course, in general, the nonzero probabilities are not all equal, but if the $n$th state has probability $p_{n}$, one can say that in some crude sense it corresponds to $N_{n}=1 / p_{n}$ states, and then the entropy is the expectation value of $\ln N_{n}$, using the probabilities $p_{n}$ as weights in averaging $\ln N_{n}$ over all states $n$.

So for a black hole, the question is what all the $\exp S_{\mathrm{bh}} \sim \exp (A / 4)$ accessible states are. Or, to put it another way, what and where are the degrees of freedom of a black hole?

One idea is that the degrees of freedom exist inside the black hole, say in the matter that has fallen in and/or in the antiparticles produced along with the particles emitted by the Hawking radiation [96, 97. This is perhaps the simplest view, but it does leave it difficult then to explain how the information about those degrees of freedom can get out when the black hole evaporates, if indeed information is not lost in black hole formation and evaporation. A possible way to resolve this difficulty is to say that quantum gravity effects dissolve the absolute distinction between the inside and the outside of the black hole, so that information that in a semiclassical approximation appears to be forever hidden inside a black hole can actually come out. Perhaps there are quantum amplitudes for wormholes from the "inside" to the outside, or perhaps just for tubular regions or conduits of trivial topology where the causal structure is sufficiently altered for the information to be conducted out [66]. It seems likely that there must be some amplitudes for such structures to occur, though the challenge would be to explain how they can funnel all of the information back out by the time that the black hole completely evaporates.

Another idea is that the degrees of freedom exist precisely on the surface of the black hole, say in its shape [58, 61]. There have been calculations from various approaches to quantum gravity that have counted the degrees of freedom of the horizon and have given (perhaps not surprisingly) an entropy proportional to the surface area [98, 99, 100, 101, 102, 103, 104, 105, 106, 107. One counter-intuitive aspect of this idea is that locally there is nothing special about the horizon (except perhaps when the geometry is eternally stationary, so that the event horizon coincides with an apparent horizon that can be located by quasi-local measurements of the geometry).

A third idea is that the important degrees of freedom are just outside the black 
hole horizon [108, 62, 109]. This is supported by calculations of the thermal atmosphere of a black hole, which is believed to have real observable effects for the buoyancy of hypothetical highly reflecting objects lowered extremely close to the black hole [71. On the other hand, the thermal atmosphere has a negligible effect on observers freely falling through the event horizon, so this makes it somewhat difficult to believe that it really would have the huge entropy that the black hole has. Another problem with ascribing the entropy to the thermal atmosphere is that a semiclassical calculation of the entropy of quantum fields outside the horizon of a classical black hole gives a divergent result, unless one puts a cutoff on the modes close to the horizon, and then the resulting entropy depends sensitively on the cutoff.

However, it seems there must be something to the argument that entropy resides in the thermal atmosphere, since if one puts in a reflecting boundary to exclude this thermal atmosphere above some height above the horizon, then the total black hole entropy is reduced below $A / 4$ by the amount one would ascribe to the thermal atmosphere that is excluded by the boundary [110]. If one could get the boundary down to within about one Planck length of the horizon, then the semiclassical calculation would say that the total entropy would be reduced to zero. Thus it is conceivable that all of the black hole entropy resides in the thermal atmosphere, but since the semiclassical approximation would break down if the boundary were placed that close to the horizon, we cannot yet be sure.

Yet another idea, or set of ideas, is that one simply cannot localize the degrees of freedom that give the black hole entropy. This would certainly seem to be the case in string/M theory, since the strings and branes that are fundamental to that theory are nonlocal objects.

For example, one of the great successes of string/M theory is giving a precise account (including Hawking's factor of $1 / 4$ in $S_{\mathrm{BH}}=A / 4$ ) of the entropy of certain kinds of black holes in terms of an extrapolation from certain D-brane configurations 111, 112, 113, 114, 115, 116, 117, 118, 119, 120, 121, 122, 123, 124, 125, 126, 127, 128, 129, 130, 131, 132, 133, 134, 135, 136, 137, 138, 139, 140, 141, 142, 143, 144, 145, 146, 147, 148, 149, 150, 151, 153, 154, 155, 156, 157, 158, 159, 160. These D-branes are nonlocal. However, it must be admitted that these calculations do not fully elucidate the nature of the nonlocality in the black hole case, because of the extrapolation needed from an understandable weak-field D-brane configuration to a strong-field black hole configuration.

A related string/M theory picture of the degrees of freedom of a black hole is that 
they reside in open strings attached to the horizon [161]. Matter falling through the horizon may be represented by closed strings approaching the black hole that become open strings attached to the horizon. Then these open strings interact strongly and eventually go back into closed strings being emitted from the horizon as Hawking radiation. Because the open strings on the horizon retain the information brought in during the black hole formation (until they are radiated back out), information is not lost in this picture. However, the strong interactions between the strings attached to the horizon mean that the information is highly scrambled or encoded, so that what is radiated can look very nearly like thermal radiation.

This is at least the view for observers that stay outside the black hole. For observers that fall through the horizon, they do not see anything special about that surface, and they see the information falling through the horizon. It would then seem that the information must have been cloned, so that a copy of what falls into the black hole is retained on its surface. However, cloning of information is forbidden in linear quantum theory [162, so this raises a puzzle. Building upon some ideas of 'tHooft [108, 163, 164, Susskind and collaborators [165, 166, 167, 168, 169, 170, 171] have proposed the principle of Black Hole Complementarity, that it doesn't matter that copies of the information have been made, since no single observer can access more than one copy.

Perhaps a related way to give an heuristic justification of Black Hole Complementarity is the following argument: In ordinary quantum field theory in a fixed classical globally hyperbolic spacetime, information cannot be cloned to appear twice on some (spatial) Cauchy hypersurface. However, unitary evolution of the quantum fields means that the same information actually does occur at different times (the same information on all Cauchy surfaces). Therefore, if one has a surface that is not everywhere spacelike, it can be connected to itself by causal curves through the spacetime and can have the same information appearing twice, say within any two regions on the surface that are connected by causal curves through the spacetime. Now in the black hole spacetime, the hypersurface where the information is supposed to appear twice (once outside the black hole in the form of Hawking radiation to be seen by an outside observer, and once inside to be seen by an infalling observer) has its normals highly boosted from one region to another, so in a sense the hypersurface becomes nearly null. Then if there are quantum uncertainties in the four-geometry, it may be indefinite whether or not the surface is really a spacelike surface and therefore whether there really is a problem with having the same information appearing 
twice on it.

That is, in quantum gravity, one would not expect a definite four-metric or even a definite causal structure, so that one cannot say with definiteness which regions are not causally related and which therefore cannot be given copies of the same information. In particular, one may never be able to say with precision that two operators in two different regions commute (or anti-commute), because one cannot say with precision that the two regions are spacelike separated (are not causally connected through the quantum spacetime). (As an aside, it would seem to me that this might lead to difficulties in canonical quantum gravity and the WheelerDeWitt equation, in which one attempts to write a quantum state as a functional of the three-geometry and matter configuration on some three-surface, which seems to assume implicitly that the local geometry and matter field variables commute for different regions of the three-surface.)

Because it seems that the degrees of freedom describing a black hole cannot be localized, and since they presumably cannot be described even in terms of some four-geometry with a definite causal structure, it may be difficult to try to give much of a description of them until we have and understand a good theory of quantum gravity.

\section{$5 \quad$ Logarithmic corrections to black hole entropy}

One area of recent developments that has not yet settled down to definitive conclusions concerns corrections to the Bekenstein-Hawking formula (2) that equates the black hole entropy $S_{\mathrm{bh}}$ to the Bekenstein-Hawking expression $S_{\mathrm{BH}} \equiv A / 4$, one-fourth the area of the event horizon. Since this formula was derived by Hawking from his semiclassical calculation of the black hole temperature, it would be expected to have quantum corrections.

One type of correction would come from the one-loop effects of quantum matter fields near a black hole. For example, Fursaev [172] calculated that with $N_{s}$ massless fields of spin $s$ present, the entropy of a Schwarzschild black hole with $S_{\mathrm{BH}} \equiv A / 4 \gg$ 1 would be

$$
S_{\mathrm{bh}}=S_{\mathrm{BH}}+\frac{1}{360}\left(4 N_{0}+7 N_{1 / 2}-52 N_{1}-233 N_{3 / 2}+848 N_{2}\right) \ln S_{\mathrm{BH}}+O(1) .
$$

Similarly, Mann and Solodukhin [173. calculated that one-loop effects of a single scalar field would modify the entropy of a large extreme Reissner-Nordstrom black 
hole to give

$$
S_{\mathrm{bh}}=S_{\mathrm{BH}}-\frac{1}{180} \ln S_{\mathrm{BH}}+O(1) .
$$

Note that for the Reissner-Nordstrom black hole, the coefficient of the logarithmic term is $-1 / 2$ what Fursaev calculated it to be for the Schwarzschild black hole with a single scalar field.

However, the main emphasis recently has been on purely quantum gravity corrections to the entropy. Kaul and Majumdar [174] performed a quantum geometry calculation that gave, again for large $S_{\mathrm{BH}}$,

$$
S_{\mathrm{bh}}=S_{\mathrm{BH}}-\frac{3}{2} \ln S_{\mathrm{BH}}+O(1) .
$$

Note that the nonperturbative calculation of Kaul and Majumdar gives the coefficient of the logarithm as -135/212 times what one would get from the result of the one-loop calculation of Fursaev with one single spin-2 particle (e.g., the graviton).

Carlip [175] reproduced the Kaul and Majumdar result from logarithmic corrections to the Cardy formula [176, 177], and Govindarajan, Kaul, and Suneeta [178] also found it from an exact expression for the partition function of the Euclidean BTZ black hole [179, 180]. Rama [181] did a calculation of the asymptotic density of open $p$-brane states and found a logarithmic term with a coefficient $-(p+1) /(2 p)$, which agrees with the Kaul-Majumdar coefficient above for open strings $(p=1)$. Gour [182] obtained the Kaul-Majumdar result for a particular approach to black hole quantization.

However, more recently there have been other calculations [183, 184, 185, 186] that have given the coefficient of the logarithm as $-1 / 2$ rather than $-3 / 2$, so that

$$
S_{\mathrm{bh}}=S_{\mathrm{BH}}-\frac{1}{2} \ln S_{\mathrm{BH}}+O(1) .
$$

Other papers on logarithmic corrections for black hole entropy include [187, 188, 189, 190, 191, 192, 193, 194, 195, 196, 197, 198, 199, 200, 201, 202, 203, 204, 205.

It should be noted that there are different ways to define the black hole entropy $S_{\mathrm{bh}}$ that can give different results [189, 193], so for any of the formulas to be meaningful, the quantities in them should be precisely defined. Eqs. (25) and (26) appear to refer to the logarithm of the number of black hole states with a certain value of the horizon area $A=4 S_{\mathrm{BH}}$, or perhaps of states with the horizon area within a certain narrow range, and so they might be called formulas for the microcanonical entropy of a black hole. Other formulas are for the von Neumann entropy,

$$
S_{\mathrm{vN}}=-\operatorname{Tr}(\rho \ln \rho)
$$


for a density matrix $\rho$ representing a quantum ensemble of black hole states, such as the canonical ensemble or the grand canonical ensemble.

Both of these definitions have certain problems associated with them.

Consider first the microcanonical ensemble, in which one generally assumes that the quantity being fixed (e.g., the horizon area) has a discrete spectrum, with each possible value having a certain degeneracy. The fine-grained microcanonical entropy would then be defined for each possible value of the quantity being fixed and would be an entropy that is the logarithm of the degeneracy. Then there seems to be two possibilities for the fine-grained microcanonical entropy:

First, it could be that all of the possible area values are nondegenerate, in which case the fine-grained microcanonical entropy would be trivially zero for each allowed value of the area or other fixed quantity. If the fixed quantity were the energy rather than the area, I would expect that nearly all of the energy eigenstates would be nondegenerate, so that in this case the fine-grained microcanonical entropy would be trivial.

(Classically, a black hole in vacuum or in the presence of a cosmological constant would have its horizon area determined by a simple function of its energy and other conserved quantities, such as angular momentum. However, when one includes quantum corrections, I would not expect the horizon area, even if it is well defined, to be determined precisely by the same simple function of the energy and other commuting conserved quantities. Even if the area and energy are both given by Hermitian operators that commute, I would not expect their precise eigenvalues to be so simply related as they are classically. Furthermore, I would not even expect both operators, if they exist, to commute, since I see no fundamental reason why a black hole configuration with definite values for the energy and other commuting conserved quantities should also have a definite value for its horizon area. Conversely, I see no reason why black holes with definite horizon areas, if such can exist, should have definite energies. Therefore, even if it is possible in the correct theory of quantum gravity to define an area operator similar to that used in the quantum geometry approach, and even if the eigenvalues of that operator are restricted to macroscopically distinct values with large degeneracies for the eigenstates with those eigenvalues, I would still think it likely that the energy eigenstates would nearly all be nondegenerate, with exponentially tiny gaps between the eigenvalues.)

Second, it could be that some or all of the possible area values are degenerate, in which case the fine-grained microcanonical entropy would be the logarithm of an 
integer (the degeneracy) for each allowed value of the area or other fixed quantity. However, in this case, I do not see any strong reason why the degeneracy, and hence the microcanonical entropy, would not vary greatly between nearby possible values of the area. For example, in quantum geometry, the allowed values of the horizon area are often taken to be proportional to an integer-weighted sum of $\sqrt{j(j+1)}$ over spins $j$ (such that $2 j$ is a positive integer), and nearby different values of these weighted sums would typically have significantly different degeneracies. An exception might be the alternative assumption that there is an equally-spaced spectrum of areas, but to me this seems to be merely an ad hoc proposal without any significant justification.

Therefore, for the microcanonical ensemble, to get a nonzero entropy that did not vary wildly between nearby values of the area or other quantity, it would seem that one would need to add up the degeneracies over a range of areas about some midpoint, or otherwise do some smoothing of the degeneracies. If the range of areas needed for the smoothing is small enough that the logarithm of the smoothed degeneracy does not vary greatly as the midpoint of the range is varied by an amount comparable to the width of the range, then the resulting smoothed microcanonical entropy would be fairly insensitive to the smoothing procedure. For very large $A=$ $4 S_{\mathrm{BH}}$, it would have an ambiguous additive constant with a linear dependence upon the logarithm of the smoothing range, so the coefficient of $\ln S_{\mathrm{BH}}$ in an expansion in powers and logarithms of $1 / S_{\mathrm{BH}}$ would depend upon how the smoothing range depends upon $S_{\mathrm{BH}}$, but it would be well defined once a definite prescription for the smoothing range is given.

However, it is not clear that this is indeed the case in the quantum geometry approach, since it would seem that one might need to smooth over a range of areas with a width that is comparable with unity (the Planck area) to suppress fluctuations in the degeneracy. But then the degeneracy summed over the range of areas would depend nonlinearly on the width of the range and on how the summed degeneracy depends on the midpoint of the range, so it might be the case that the coefficient of the logarithmic term in the smoothed microcanonical entropy would have a more significant dependence upon the smoothing procedure. Maybe it would not, but that would remain to be checked.

Another problem I see with the microcanonical entropy is that at present it is being calculated with the horizon area fixed, but it would seem to me that that quantity would be difficult to measure. Although quantum geometry gives an operator for it, I am not convinced that it would be a well-defined quantity in other 
approaches to quantum gravity (e.g., in string/M theory).

It would seem to me much better to use something like the total energy as the quantity to be fixed in a microcanonical ensemble. This would require appropriate asymptotic boundary conditions, such as asymptotically anti-de Sitter boundary conditions with reflecting boundary conditions at infinity [206]. It would not work in asymptotically flat spacetime, since then the infinite volume of phase space at any finite energy would mean that it is entropically favorable for the black hole to evaporate away, and so the microcanonical ensemble would be dominated by diffuse radiation states rather than by black hole states.

Even in asymptotically anti-de Sitter spacetime, small black holes would decay away, so for black holes to dominate the microcanonical ensemble, one needs the total energy $E$ to be large enough that the black hole can be in stable equilibrium with the Hawking radiation surrounding it. In $d=4$ dimensions of spacetime, the condition for black holes to dominate the microcanonical ensemble is given by Eq. (4.14) of [206], except that there is no upper limit for the energy if one does not also ask, as we did there, that pure thermal radiation without a black hole also be a local equilibrium state.

In a general dimension $d$ of spacetime, with $\ell \gg 1$ being the length scale of the anti-de Sitter spacetime in Planck units (the length we called $b$ in [206]), a black hole with horizon radius $r_{+} \ll \ell$ would have a mass $M \sim r_{+}^{d-3}$, a temperature $T \sim 1 / r_{+}$, and an entropy $S_{\mathrm{bh}} \sim r_{+}^{d-2} \sim M^{(d-2) /(d-3)}$ in Planck units, omitting the dimensiondependent numerical coefficients that would be of order unity for dimensions of order unity. The black hole would be surrounded by thermal Hawking radiation of massless fields with energy density $\rho \sim T^{d}$ and entropy density $s \sim T^{d-1}$ out to $r \sim \ell$, and then with the rapidly rising anti-de Sitter gravitational potential causing the local redshifted temperature, energy density, and entropy density all to drop off exponentially with proper distance so that the total radiation energy $E_{\mathrm{rad}} \sim \ell^{d-1} T^{d}$ and the total radiation entropy $S_{\mathrm{rad}} \sim \ell^{d-1} T^{d-1} \sim\left(\ell E_{\mathrm{rad}}\right)^{1-1 / d}$ are finite, even when one integrates over the infinite proper spatial volume of the anti-de Sitter space. (This ignores the divergence one gets in integrating locally thermal radiation down to the black hole horizon, where the local temperature blueshifts to infinity. Alternatively, one can assume that this divergence at the horizon is regulated in some manner.)

Now the idea [37, 207, 206] is that for fixed total energy $E \approx M+E_{\text {rad }}$, the equilibrium configuration has the energy divided between the black hole mass $M$ 
and the radiation energy $E_{\mathrm{rad}}$ in such a way to maximize the total entropy $S=$ $S_{\mathrm{bh}}+S_{\mathrm{rad}} \sim M^{(d-2) /(d-3)}+[\ell(E-M)]^{(d-1) / d}$. (I am ignoring the interaction energy between the black hole and the radiation, which would be small if the black hole is small and hence takes up only a tiny fraction of the volume available to the radiation where the gravitational potential is not too large. However, it would not necessarily be negligible if one were trying to calculate all terms in the total entropy $S$ that are large in comparison with unity, so that one can get the number of states, $\sim e^{S}$, correct with a fractional error of the order of unity.)

If $E$ is too small, the only maximum of the entropy $S(M)$ would be at $M=0$, meaning that pure thermal radiation with no black hole would dominate the microcanonical ensemble. For $E$ larger than some energy $E_{0}$ that is some dimensionless number $c_{0}$ (depending on the number $d$ of spacetime dimensions and on the radiation constant that depends on the number and types of massless fields) times $\ell^{(d-1)(d-3) /(2 d-3)}$, two new extrema of $S(M)$ appear at positive $M$, where the black hole temperature is the same at that of the surrounding radiation. When one perturbs either extremum by making the black hole mass smaller and the radiation energy larger, both the black hole and the surrounding radiation get hotter (since the black hole with $r_{+} \ll \ell$ has negative specific heat, and since the radiation has positive specific heat).

However, for the extremum with smaller positive $M$, when one perturbs to yet smaller $M$, the black hole temperature rises more than the surrounding radiation, so the black hole stays hotter than the surrounding radiation and is unstable to evaporating away. Alternatively, if the black hole mass $M$ is perturbed to a larger value than the extremum, the black hole temperature cools more than the surrounding radiation, so then the black hole is unstable to absorbing yet more radiation and continuing to grow. This extremum is thus a local minimum of $S(M)$ and represents unstable equilibrium between a black hole and surrounding radiation with the same temperature.

On the other hand, for the extremum with larger positive $M$, when one perturbs $M$ to a smaller value, the black hole temperature rises less than the temperature of the radiation, so the radiation gives a negative feedback on the shrinkage of the hole, keeping it stable. Alternatively, if the black hole mass $M$ is perturbed to a larger value than the extremum, the black hole temperature cools less than the surrounding radiation, so then the black hole is hotter than the surrounding radiation and thus emits its excess energy to return to its equilibrium mass. This extremum is thus a 
local maximum of $S(M)$ and represents a locally stable equilibrium between a black hole and surrounding radiation with the same temperature.

Note that if one started with a black hole at the unstable extremum (the smaller positive value of $M$ that gives an extremum) and then perturbed $M$ to a larger value, at first the black hole would have an unstable growth as described above, but eventually it would settle down at the locally stable extremum with the larger value of $M$. On the other hand, if one perturbed the unstable equilibrium to give $M$ a smaller value, the black hole would continue to shrink until it disappeared, giving the other locally stable configuration, pure thermal radiation. It would require a nonperturbative fluctuation to transit between these two locally stable configurations.

Since the Hawking temperature for a black hole much smaller than the characteristic length scale $\ell$ of the anti-de Sitter spacetime of $d$ spacetime dimensions (black hole horizon radius $\left.r_{+} \ll \ell\right)$ is proportional to $M^{-1 /(d-3)}$, and since the temperature of thermal radiation in $d$ dimensions is proportional to $E_{\mathrm{rad}}^{1 / d}$, one can readily see that the condition for the black hole temperature to rise less than the radiation when a small amount of energy is transferred from the black hole (reducing $M$ ) to the radiation (increasing $E_{\text {rad }} \approx E-M$ by the essentially the same amount), starting at the condition when the two temperatures are equal, is that $E_{\mathrm{rad}}<(1-3 / d) M$, or $E_{\text {rad }}<[(d-3) /(2 d-3)] E$, or $M>[d /(2 d-3)] E$. When one saturates these inequalities and requires that the black hole and radiation temperatures be equal, one gets $E=E_{0}=c_{0} \ell^{(d-1)(d-3) /(2 d-3)}$ and can evaluate the numerical coefficient $c_{0}$ for various spacetime dimensions and values of the radiation constant [37, 207, 206].

Therefore, for $E>E_{0}$, there is a locally stable equilibrium microcanonical configuration with a black hole having positive mass $M$. However, the pure thermal radiation configuration with $M=0$ also remains a locally stable equilibrium microcanonical configuration, at least for $E<E_{2}=c_{2} \ell^{d-3} \sim \ell^{d-3} \gg E_{0}$ with some numerical coefficient depending purely upon $d$ (assuming that the radiation is purely massless radiation). (For $E>E_{2}$, any configuration of pure radiation would be dynamically unstable to collapsing to form a black hole, e.g., by the Jeans instability.) Which of the two locally stable configurations will dominate the microcanonical ensemble depends upon their entropies.

For $E_{0}<E<E_{1}=c_{1} \ell^{(d-1)(d-3) /(2 d-3)}$ with another numerical coefficient $c_{1}$ that is somewhat larger than $c_{0}$ (e.g., larger by a factor of about 1.314 for $d=4$ [206]), the pure thermal radiation configuration has more entropy and so dominates. But 
for $E>E_{1}$, the configuration with a black hole in locally stable thermal equilibrium with surrounding radiation having less energy than $[(d-3) / d] M$ has more entropy and so dominates the microcanonical ensemble.

Therefore, we can say that for $E>E_{1} \sim \ell^{(d-1)(d-3) /(2 d-3)}$, black hole configurations dominate the microcanonical ensemble of fixed energy $E$ in asymptotically anti-de Sitter spacetime.

One would expect that in a quantum analysis, one would get a discrete set of energy eigenvalues. In principle, from sufficient (future) knowledge of the quantum theory of gravity and matter in asymptotically anti-de Sitter spacetime with length scale $\ell$, one could count the number $N(E)$ of energy eigenstates up to any energy $E$, and then one could use this to define various microcanonical entropies. Again one would face the challenge that the fine-scale microcanonical entropy, the logarithm of the degeneracy of a precise energy eigenvalue, is likely either to be zero (if the energy eigenvalues are nondegenerate, as I would expect, unless perhaps $\ell$ can be tuned to give a degeneracy) or else fluctuate between nearby energy eigenvalues (if various ones are degenerate).

Therefore, it seems likely that some smoothing over nearby energy eigenvalues would be needed. However, since for $E \gg E_{1}$ a semiclassical analysis, using the Bekenstein-Hawking entropy for the dominant black hole with $M \approx E$, suggests that, very crudely, $N(E) \sim \exp \left[c E^{(d-2) /(d-3)}\right]$ for some readily-calculable numerical coefficient $c$ that depends purely upon $d$. Thus $N(E)$ rises very rapidly with $E$ (faster than exponentially with the energy). Unless the energy eigenvalues are clumped into values with huge degeneracies that are then separated by macroscopic amounts (e.g., by amounts comparable with the black hole temperature), which seems very implausible to me, one would expect a huge number of eigenvalues within any range of energies that is not extraordinarily narrow. Therefore, one could do a fairly generic smoothing over any range of energies (but a range large enough to contain a huge number of energy eigenvalues) to get a coarse-grained microcanonical entropy.

However, there would remain some ambiguities in the coarse-grained microcanonical entropy, depending upon how the range of energies was defined. One of the least ad hoc definitions would be to define

$$
S_{\text {microcanonical }}(E)=\ln N(E) \text {, }
$$

where $N(E)$ counts all of the energy eigenstates up to energy $E$, so then the range would go all the way from the minimum energy state to $E$. 
Nevertheless, one could alternatively define coarse-grained microcanonical entropies with narrower ranges, say

$$
S_{\text {microcanonical }}^{\prime}(E)=\ln [N(E+\Delta)-N(E-\Delta)]
$$

the logarithm of the number of energy eigenstates with energy within $\Delta$ of $E$. If $N(E+\Delta)-N(E-\Delta) \ll N(E-\Delta)$, which for the rapidly growing $N(E)$ implies $\Delta \ll E$ but is much stronger than that condition, $S_{\text {microcanonical }}^{\prime}(E)$ would have a roughly linear dependence upon the logarithm of $\Delta / E$.

For example, if for the sake of argument one assumed that $N(E)=\exp \left(c E^{\alpha}\right)$ for some constants $c$ and $\alpha$ (e.g., for $\alpha=(d-2) /(d-3)$ ), here and henceforth neglecting the truncation to the largest integer not greater than $\exp \left(c E^{\alpha}\right)$ that would be needed for $N(E)$ to be an integer, then

$$
S \equiv S_{\text {microcanonical }}(E)=\ln N(E)=c E^{\alpha}
$$

whereas for $c \alpha E^{\alpha-1} \Delta \ll 1$ so that $N(E+\Delta)-N(E-\Delta) \ll N(E-\Delta)$, one gets

$$
\begin{aligned}
S^{\prime} & \equiv S_{\text {microcanonical }}^{\prime}(E)=\ln [N(E+\Delta)-N(E-\Delta)] \\
& \approx \ln \left[e^{c E^{\alpha}}\left(e^{c \alpha E^{\alpha-1} \Delta}-e^{-c \alpha E^{\alpha-1} \Delta}\right)\right] \approx \ln \left[e^{c E^{\alpha}} 2 c \alpha E^{\alpha-1} \Delta\right] \\
& =S+\ln S+\ln (\Delta / E)+\ln (2 \alpha) .
\end{aligned}
$$

This example shows how logarithms can come in when one goes from one coarse graining to another. Here the coefficient of the logarithm of the energy depends on how the width of the range depends on the energy. For example, if we set $\Delta=\beta E^{\gamma}$, then

$$
S^{\prime}=S+(\alpha+\gamma-1) \ln E+\ln (2 \alpha \beta c)=S+\frac{\alpha+\gamma-1}{\alpha} \ln S+\ln (2 \alpha \beta)-\frac{\gamma-1}{\alpha} \ln c .
$$

Thus by choosing $\gamma$ appropriately, one can get any coefficient of the logarithm term that one wants. In this case it would be absent if $\gamma=1-\alpha$, which would be the case if the range were made to give equal widths for the classical horizon area $A \propto E^{\alpha}$, rather than equal widths for any other power of $E$.

Thus we see that the coefficient of a logarithm term in an entropy does not have any meaning unless one carefully specifies how the entropy is to be defined.

What would be more meaningful would be a difference between an entropy calculated classically (or semiclassically) and one calculated with the full theory of quantum gravity and some specific definition of the entropy. Ideally, one would like 
both entropies to be defined the same way, but since a classical or semiclassical calculation is not likely to lead to discrete energy eigenstates, there may not be a precise classical or semiclassical analogue for many of the definitions of entropy possible in the quantum theory with definite discrete energy eigenstates.

The classical or semiclassical calculation would be easiest if one fixed the black hole area, or a range of areas, since the classical Bekenstein-Hawking formula for the entropy is simply one-fourth the area. However, as mentioned above, for a comparison with a quantum theory, it would probably be better to do a comparison in terms of a quantity like the energy that is more likely to be well defined in the quantum theory (under suitable conditions). For example, in string theory on an asymptotically anti-de Sitter background, presumably the energy would be well defined, but it may be doubtful whether any horizon or any horizon area is well defined.

If one used energy rather than horizon area as the quantity that may be well defined both classically and quantum mechanically, then one would like to compare some quantum entropy with a classical calculation for it. For example, one might take the classical entropy to be the Bekenstein-Hawking entropy $S_{\mathrm{BH}} \equiv A / 4$ for a classical configuration with the same energy $E$.

However, if the semiclassical description is of a black hole surrounded by thermal radiation, one would expect the black hole entropy to be augmented by the entropy of the radiation (at least the part of the radiation far from the hole, if the black hole entropy includes the effects of radiation very near the horizon). One might want to include some approximation for this entropy within the semiclassical entropy. But then it becomes a question of precisely how this is to be done within the semiclassical approximation, if one wants to compare it with the quantum result (once that is known, with some suitable definition of the quantum entropy from the spectrum of energy eigenstates).

For example, above I alluded to an approximation in which one took a small black hole in the vacuum Schwarzschild-anti-de Sitter spacetime (which classically has well-defined relations between the black hole horizon radius $r_{+}$, the mass $M$, the temperature $T$, and the Bekenstein-Hawking entropy $S_{\mathrm{BH}} \equiv A / 4$ for a given anti-de Sitter length scale $\ell$ ) and then just added on the energy and entropy of thermal radiation in anti-de Sitter spacetime (for this ignoring the change in the geometry from the black hole).

Even for the latter energy and entropy, there is some ambiguity on how it is to 
be calculated classically or semiclassically. The simplest approximation would be to assume that the massless radiation had local energy and entropy densities given by the flat spacetime radiation constant multiplied by the appropriate dimensiondependent powers of the local temperature, and to neglect the back reaction of the radiation on the geometry. A slightly improved calculation would be to take the quantum canonical ensemble of the massless radiation fields in the fixed anti-de Sitter background and calculate from it the expectation value of the energy and the von Neumann entropy. A further improved but difficult calculation would be to take the quantum canonical ensemble for the radiation fields in a static classical asymptotically anti-de Sitter background (with no black hole at this stage) that solves the Einstein equation with the cosmological constant and the expectation value of the stress-energy tensor of the quantum fields in their canonical ensemble.

However, when one has both the black hole and the radiation, one would think that the semiclassical calculation should be improved by including the effect of the black hole on the asymptotically anti-de Sitter metric where the thermal radiation resides. A classical way to attempt to do this would be to take the Schwarzschildanti-de Sitter metric for the black hole with the negative cosmological constant and assume that the radiation is described by a traceless stress-energy tensor that has isotropic pressures and an energy density given by the flat spacetime radiation constant multiplied by the appropriate power of the local Hawking temperature. But this would lead to a divergence of the energy and entropy densities of the radiation at the black hole horizon, where the local Hawking temperature diverges. Therefore, it would be better (though more difficult) to take the canonical ensemble for the quantum radiation field in the Schwarzschild-anti-de Sitter metric, with the divergence at the horizon regulated. An even better but much more difficult calculation would be to take the quantum canonical ensemble for the radiation fields in a static classical asymptotically anti-de Sitter background with the black hole present that is solves the Einstein equation with the cosmological constant and the expectation value of the stress-energy tensor of the quantum fields in their canonical ensemble. If this gives an unambiguous entropy for the quantum fields, one could add on one-quarter of the black hole area to get a semiclassical entropy as a function of the ADM energy of the configuration.

Then one could compare this semiclassical result with the full quantum gravity result once that is known. However, the later will depend on how the entropy is defined, so it still would not be unambiguous what difference quantum gravity 
makes to the entropy. But at least one would presumably get a definite result for each possible definition of the quantum entropy.

If one is just looking for a difference involving the logarithm of the BekensteinHawking entropy $S_{\mathrm{BH}} \equiv A / 4$, one would not need the precise semiclassical or quantum entropy, but only the terms that involve positive powers or logarithms of $S_{\mathrm{BH}}$. However, even this might be difficult for the semiclassical entropy.

For example, if we consider the microcanonical ensemble discussed above when $E$ is comparable with $E_{1}$, one could presumably write the semiclassical entropy as a power series (perhaps including logarithms) in the small classical quantity $r_{+} / \ell$, the linear size of the horizon divided by the linear characteristic size of the asymptotically anti-de Sitter spacetime. However, for $E \sim E_{1} \sim \ell^{(d-1)(d-3) /(2 d-3)}$, one has $r_{+} / \ell \sim \ell^{-(d-2) /(2 d-3)}$, and the leading classical term in the entropy goes as $E^{(d-2) /(d-3)} \sim r_{+}^{d-2} \sim \ell^{(d-1)(d-2) /(2 d-3)} \sim\left(r_{+} / \ell\right)^{-(d-1)}$. Therefore, if this leading term is multiplied by a correction factor that is an increasing power series in $r_{+} / \ell$ (starting with unity), and if all positive powers occurred, one would need the leading classical term and $d-1$ others to include all terms in the entropy that are not small for small $r_{+} / \ell$. (The term that is zero-order in $r_{+} / \ell$, in the product of the leading term and the correction factor power series, might be a logarithmic term.) Thus we might need to calculate $d$ terms in a power series to determine the semiclassical entropy (as a function of the ADM mass) to enough precision to identify a difference with the quantum entropy (once that more difficult calculation can be performed) that is logarithmic in $S_{\mathrm{BH}}$.

For example, there could be terms in the power series in $r_{+} / \ell$ that would express the effect of the black hole on the radiation, the self-gravitational effects of the radiation itself, departures of the expectation value of the stress-energy tensor of the radiation from having an isotropic pressure, interactions between these effects, etc.

One way to circumvent some of these problems with the semiclassical treatment of the radiation would be to take a black hole in asymptotically anti-de Sitter spacetime that is very large in comparison with the anti-de Sitter length scale, so $r_{+} \gg \ell$. Then the exponentially rising gravitational potential at distances much greater than $\ell$ from the black hole would suppress the thermal radiation so that it would have very little energy and entropy in comparison with that of the hole (at least if one regulated the divergence at the black hole horizon). Then in the limit that $r_{+} / \ell$ is made very large (and also $\ell$ is kept large in Planck units to keep the geometry 
nearly classical), one might be able to ignore the effects of radiation altogether on the semiclassical calculation of the energy and entropy and simply take their values from the Schwarzschild-anti-de Sitter metric and the Bekenstein-Hawking entropy. It would be the relationship between these two quantities that one could use to compare with the quantum entropy.

Another advantage of using $r_{+} \gg \ell$ is that then not only the microcanonical ensemble but also the canonical ensemble is well defined in asymptotically anti-de Sitter boundary conditions [206]. (In asymptotically flat spacetime, the canonical ensemble is not defined, because the number of states of a black hole rises indefinitely faster than exponentially with the energy [37.) Therefore, not only is there the natural coarse-grained microcanonical entropy defined by Eq. (30), the logarithm of the number of energy eigenstates with energy below $E, S_{\text {microcanonical }}(E)=\ln N(E)$, but there is also the canonical entropy that may be defined to be a function of the energy expectation value $\langle E\rangle$ :

$$
S_{\text {canonical }}(\langle E\rangle)=\beta\langle E\rangle+\ln Z
$$

where the partition function is

$$
Z(\beta)=\sum_{i} e^{-\beta E_{i}}
$$

with the index $i$ running over all energy eigenstates with energy $E_{i}$ (more than one of which could be the same if any energy eigenvalues are degenerate), and where

$$
\langle E\rangle=-\frac{\partial}{\partial \beta} \ln Z
$$

denotes the expectation value of the energy.

For energies such that $N(E)$ is very large, one can approximate the discrete function $N(E)$ (taking only integer values) with a continuous function, say $n(E)=$ $e^{s(E)}$ with $s(E)$ being some continuum approximation to $S_{\text {microcanonical }}(E)=\ln N(E)$. Then the sum above for the partition function $Z(\beta)$ would be replaced by the integral

$$
Z(\beta) \approx z(\beta)=\int e^{-\beta E} d n=\int e^{s-\beta E(s)} d s
$$

where $E(s)$ is the inverse function to $s(E)=\ln n(E)$.

Now define the auxiliary function $T(s) \equiv d E / d s$ (a microcanonical temperature) and assume that it is always positive and, at least for all $s$ above some value $s_{0}$ where it has a local minimum value $T_{0}$, that $T(s)$ rises smoothly, monotonically, and 
indefinitely with $s$. This implies that for $s>s_{0}$, the exponent in the integral, $I(s)=$ $s-\beta E(s)$, is concave downward, with $d I / d s=1-\beta T(s)$ decreasing monotonically with $s$. Therefore, for any fixed $\beta<1 / T_{0}$, there will be a unique extremum of $I(s)$, at the unique value of $s=s(\beta)>s_{0}$ where $T(s(\beta))=1 / \beta$. Let this extremum value of $I(s)$ be denoted $I_{\max }(\beta)=s(\beta)-\beta E(\beta)$, with $E(\beta)=E(s(\beta))$ being the value of $E$ at $s=s(\beta)$.

It is also convenient to define the microcanonical heat capacity function $C(s)=$ $d E / d T$, which will be positive for $s>s_{0}$, since then both $E$ and $T$ are rising monotonically with $s$. Let its value at the extremum value of $I(s)$ be $C(\beta)=$ $C(s(\beta))$. Since one can readily show that $d^{2} I(s) / d s^{2}=-1 / C(s)$, for $s$ sufficiently near the value $s(\beta)$ that extremizes $I(s)$, one has

$$
I(s) \approx I_{\max }(\beta)-\frac{1}{2 C(\beta)}[s-s(\beta)]^{2} .
$$

If this approximation is valid over the dominant part of the integral (36) for $z(\beta)$, one can do the integral by the saddle-point approximation and get the value

$$
Z(\beta) \approx z(\beta) \approx \sqrt{2 \pi C(\beta)} e^{I_{\max }(\beta)}
$$

Then one can use Eqs. (35) and (33) to calculate

$$
\langle E\rangle \approx E(\beta)+\frac{T^{2}}{2 C} \frac{d C}{d T}
$$

where $T$ and $C$ and $d C / d T$ are evaluated at $s=s(\beta)$, and to calculate

$$
S_{\text {canonical }} \approx s+\frac{1}{2} \ln (2 \pi C)+\frac{T}{2 C} \frac{d C}{d T}
$$

where again everything is evaluated at $s=s(\beta)$.

For a large black hole in anti-de Sitter spacetime, asymptotically at large $r_{+} / \ell$ one has $E \approx M \sim r_{+}^{d-1} / \ell^{2}$ and $s \approx S \sim r_{+}^{d-2} \sim E^{\alpha}$ with $\alpha=(d-2) /(d-1)$, so $E(s) \sim s^{(d-1) /(d-2)}, T(s)=d E / d s \sim s^{1 /(d-2)}$, and $C(s)=d E / d T \approx(d-2) s$. Therefore, one gets

$$
S_{\text {canonical }} \approx s+\frac{1}{2} \ln s+O(1)
$$

Remembering that $s$ is a continuum approximation to $S_{\text {microcanonical, one gets that }}$ when either entropy is large, these two entropies differ mainly by the logarithm of the entropy (dropping terms of the order of unity):

$$
S_{\text {canonical }} \approx S_{\text {microcanonical }}+\frac{1}{2} \ln S_{\text {microcanonical }}
$$




$$
S_{\text {microcanonical }} \approx S_{\text {canonical }}-\frac{1}{2} \ln S_{\text {canonical }} .
$$

Similar relations have been given in [189, 193, though they used a microcanonical entropy defined as the logarithm of the density of states as a function of energy, rather than my use of the microcanonical entropy as the logarithm of the total number of states of lower energy. From Eqs. (31) and (32) above, one can see that one can get further changes in the coefficient of the logarithm by going to a microcanonical entropy defined as the logarithm of the number of states within some range of energies, depending on how the range varies with the midpoint of the range.

Therefore, entropies need to be defined carefully before there is any unambiguous meaning to logarithmic corrections. But even if one does choose a precise definition, it may be a long time before we have sufficient knowledge of quantum gravity to be able to calculate the correct answers for the logarithmic terms in the entropy.

\section{Conclusions}

Black holes are perhaps the most highly thermal objects in the universe (though they are very cold for stellar mass black holes). Their phenomenological thermodynamic properties are very well understood (at least for quasistationary semiclassical black holes), but a good understanding of their microscopic degrees of freedom is lacking. Although it seems that black holes are rather like other thermal objects (say in having such degrees of freedom that carry the information imparted into them and restore this information to the outer universe when the black holes evaporate away), one is not yet completely sure that this is the case, or, if it is, where and how the microscopic degrees of freedom store the information. Therefore, although we have gained an enormous amount of information about black holes and their thermal properties in the past thirty years, it seems that there is even much more that we have yet to learn.

Spacetime limitations on the author have prevented this review from being anywhere near complete. For other recent (and often more nearly complete) reviews, see [208, 209, 61, 210, 211, 212, 213, 214, 215, 216, 217, 218, 219, 220, 221, 222, 223, 224].

\section{Acknowledgments}

I am grateful for many discussions with, among others, Abhay Ashtekar, Tom Banks, Andrei Barvinsky, Jacob Bekenstein, David Boulware, Raphael Bousso, Steve Car- 
lip, Brandon Carter, Paul Davies, David Deutsch, Bryce DeWitt, Doug Eardley, Richard Feynman, Willy Fischler, Eanna Flanagan, Larry Ford, Valeri Frolov, Ulrich Gerlach, Gary Gibbons, Jim Hartle, Stephen Hawking, Gerard 't Hooft, Gary Horowitz, Werner Israel, Juan Maldacena, Robb Mann, Don Marolf, Charlie Misner, Rob Myers, Ted Newman, Igor Novikov, Leonard Parker, Amanda Peet, Roger Penrose, Joe Polchinski, Chris Pope, Bill Press, Richard Price, Rafael Sorkin, Mark Srednicki, Alexie Starobinsky, Andy Strominger, Lenny Susskind, Saul Teukolsky, Kip Thorne, Bill Unruh, Cumrun Vafa, Bob Wald, John Wheeler, Ed Witten, Bill Wootters, Yakov Zel'dovich, Andrei Zelnikov, and Wojciech Zurek. I am grateful for referees for pointing out the relevance of references [11, 12, 13, 14, 15, 16] and for encouraging me to include a discussion of logarithmic corrections to the entropy of a black hole. Financial support has been provided by the Natural Sciences and Engineering Research Council of Canada.

\section{References}

[1] J. M. Bardeen, B. Carter, and S. W. Hawking, Comm. Math. Phys. 31, 161 (1973).

[2] B. Carter, in C. DeWitt and B. S. DeWitt, eds., Black Holes (Gordon and Breach, New York, 1973), p. 57.

[3] S. W. Hawking, Phys. Rev. Lett. 26, 1344 (1971).

[4] W. Israel, Phys. Rev. Lett. 57, 397 (1971).

[5] J. D. Bekenstein, Ph.D. thesis, Princeton University (1972).

[6] J. D. Bekenstein, Lett. Nuovo Cimento 4, 737 (1972).

[7] J. D. Bekenstein, Phys. Rev. D7, 2333 (1973).

[8] J. D. Bekenstein, Phys. Rev. D9, 3292 (1974).

[9] S. W. Hawking, Nature 248, 30 (1974).

[10] S. W. Hawking, Comm. Math. Phys. 43, 199 (1975).

[11] L. Parker, Ph.D. thesis, Harvard University (1966).

[12] L. Parker, Phys. Rev. Lett. 21, 562 (1968).

[13] L. Parker, Phys. Rev. 183, 1057 (1969).

[14] L. Parker, Phys. Rev. D3, 346 (1971). 
[15] S. A. Fulling, Ph.D. thesis, Princeton University (1972).

[16] S. A. Fulling, Phys. Rev. D7, 2850 (1973).

[17] N. N. Bogoliubov, V. V. Tolmachev, and D. V. Shirkov, A New Method in the Theory of Superconductivity (Consultants Bureau, New York, 1959). I cannot resist the temptation to use the fact that the name "Bogoliubov" is the Russian equivalent of "Amadeus" and of "Theophilus" (see Luke 1:3), to coin the pun that Christian conversion is a Bogoliubov transformation.

[18] D. N. Page, Ph.D. thesis, California Institute of Technology (1976).

[19] Ya. B. Zel'dovich, ZhETF Pis. Red. 14, 270 (1970); English translation in Sov. Phys. JETP Lett. 14, 180.

[20] Ya. B. Zel'dovich, Zh. Eksp. Teor. Fiz. 62, 2076 (1972); English translation in Sov. Phys. JETP 35, 1085.

[21] C. W. Misner, Bull. Am. Phys. Soc. 17, 472 (1972).

[22] A. A. Starobinsky, Zh. Eksp. Teor. Fiz. 64, 48; English translation in Sov. Phys. JETP 37, 28 (1973).

[23] J. D. Bekenstein, Phys. Rev. D7, 949 (1973).

[24] W. G. Unruh, Phys. Rev. Lett. 31, 1265 (1973).

[25] W. Pauli, Phys. Rev. 58, 716 (1940).

[26] J. D. Bjorken and S. D. Drell, Relativistic Quantum Mechanics (New York, McGraw-Hill, 1964), p. 42.

[27] A. I. Nikishov, Nucl. Phys. B21, 346 (1970).

[28] W. G. Unruh, Phys. Rev. D10, 3194 (1974).

[29] L. H. Ford, Phys. Rev. D12, 2963 (1975).

[30] D. G. Boulware, Phys. Rev. D13, 2169 (1976).

[31] P. C. W. Davies, "On the Origin of Black Hole Evaporation Radiation," King's College London preprint (1976).

[32] U. H. Gerlach, Phys. Rev. D14, 1479 (1976).

[33] B. S. DeWitt, Phys. Rep. 19C, 295 (1975).

[34] W. G. Unruh, Phys. Rev. D14, 870 (1976).

[35] R. M. Wald, Comm. Math. Phys. 45, 9 (1975).

[36] L. Parker, Phys. Rev. D12, 1519 (1975).

[37] S. W. Hawking, Phys. Rev. D13, 191 (1976). 
[38] J. D. Bekenstein, Phys. Rev. D12, 3077 (1975).

[39] J. B. Hartle and S. W. Hawking, Phys. Rev. D13, 2188 (1976).

[40] S. W. Hawking, Sci. Am. 236, 34 (1977).

[41] J. D. Bekenstein, Phys. Today 33, 24 (1980).

[42] J. A. Wheeler, A Journey into Gravity and Spacetime (W. H. Freeman \& Co., New York, 1990).

[43] R. P. Kerr, Phys. Rev. Lett. 11, 237 (1963).

[44] E. T. Newman, E. Couch, K. Chinnapared, A. Exton, A. Prakash, and R. Torrence, J. Math. Phys. 6, 918 (1965).

[45] W. Israel, Phys. Rev. 164, 1776 (1967).

[46] B. Carter, Phys. Rev. Lett. 26, 331 (1971).

[47] S. W. Hawking and G. F. R. Ellis, The Large Scale Structure of Space-Time (Cambridge University Press, Cambridge, 1973).

[48] D. C. Robinson, Phys. Rev. Lett. 34, 905 (1975).

[49] P. O. Mazur, J. Phys. A15, 3173 (1982); see also hep-th/0101012 for a recent review of black hole uniqueness theorems.

[50] D. N. Page, Phys. Rev. D13, 198 (1976).

[51] F. P. Pijpers, Mon. Not. Roy. Astron. Soc. 297, L76 (1998).

[52] D. N. Page, Class. Quant. Grav. 151669 (1998).

[53] D. N. Page, Phys. Rev. D14, 3260 (1976).

[54] D. N. Page, Phys. Rev. Lett. 50, 1013 (1983).

[55] W. H. Zurek and K. S. Thorne, Phys. Rev. Lett. 54, 2171 (1985).

[56] K. S. Thorne, W. H. Zurek, and R. H. Price, in K. S. Thorne, R. H. Price, and D. A. MacDonald, eds., Black Holes: The Membrane Paradigm (Yale University Press, New Haven, 1986), p. 280.

[57] V. P. Frolov and D. N. Page, Phys. Rev. Lett. 71, 3902 (1993).

[58] R. D. Sorkin, in B. Bertotti, F. de Felice, and A. Pascolini, eds., Tenth International Conference on General Relativity and Gravitation, Padua, 4-9 July, 1983, Contributed Papers, vol. II (Roma, Consiglio Nazionale Delle Ricerche, 1983), p. 734 .

[59] R. D. Sorkin, Phys. Rev. Lett 56, 1885 (1986). 
[60] R. M. Wald, Quantum Field Theory in Curved Spacetime and Black Hole Thermodynamics (University of Chicago Press, Chicago, 1994).

[61] R. D. Sorkin, in R. M. Wald, ed., Black Holes and Relativistic Stars (University of Chicago Press, Chicago, 1998), p. 177; gr-qc/9705006.

[62] L. Bombelli, R. K. Koul, J. Lee, and R. Sorkin, Phys. Rev. D34, 373 (1986).

[63] M. Srednicki, Phys. Rev. Lett. 71, 666 (1993).

[64] S. W. Hawking, Phys. Rev. D14, 2460 (1976).

[65] D. N. Page, Phys. Rev. Lett. 44, 301 (1980).

[66] For a long review with 292 references, see D. N. Page, in R. B. Mann and R. G. McLenaghan, eds., Proceedings of the 5th Canadian Conference on General Relativity and Relativistic Astrophysics (World Scientific, Singapore, 1994), p. $1 ;$ hep-th/9305040.

[67] S. W. Hawking, "The information paradox for black holes," 17th International Conference on General Relativity and Gravitation, Dublin, Ireland (2004 July 21); see http://math.ucr.edu/home/baez/week207.html for a transcript.

[68] J. D. Bekenstein, Phys. Rev. D23, 287 (1981).

[69] S. D. Unwin, Phys. Rev. D26, 944 (1982).

[70] D. N. Page, Phys. Rev. D26, 947 (1982).

[71] W. G. Unruh and R. M. Wald, Phys. Rev. D25, 942 (1982).

[72] D. Deutsch, Phys. Rev. Lett. 48, 286 (1982).

[73] W. G. Unruh and R. M. Wald, Phys. Rev. D27, 2271 (1983).

[74] O. B. Zaslavskii, Class. Quant. Grav. 13, L7 (1996).

[75] M. A. Pelath and R. M. Wald, Phys. Rev. D60, 104009 (1999).

[76] D. N. Page, "Huge violations of Bekenstein's entropy bound," gc-qc/0005111.

[77] D. N. Page, "Subsystem entropy exceeding Bekenstein's bound," hep-th/0007237.

[78] D. N. Page, "Defining entropy bounds," hep-th/0007238.

[79] D. Marolf and R. D. Sorkin, Phys. Rev. D69, 024014 (2004).

[80] J. D. Bekenstein, Phys. Rev. D27, 2262 (1983).

[81] J. D. Bekenstein, Phys. Rev. D49, 1912 (1994).

[82] J. D. Bekenstein, Phys. Rev. D60, 124010 (1999). 
[83] J. D. Bekenstein, "On Page's examples challenging the entropy bound," gr-qc/0006003.

[84] J. D. Bekenstein, "How does the entropy/information bound work?" quant-ph/0404042.

[85] R. Bousso, J. High Energy Phys. 9907, 004 (1999).

[86] R. Bousso, Class. Quant. Grav. 17, 997 (2000).

[87] E. E. Flanagan, D. Marolf, and R. M. Wald, Phys. Rev. D62, 084035 (2000).

[88] R. Bousso, Rev. Mod. Phys. 74, 825 (2002).

[89] R. Bousso, E. E. Flanagan, and D. Marolf, Phys. Rev. D68, 064001 (2003).

[90] R. Bousso, J. High Energy Phys. 0104, 035 (2001).

[91] R. Bousso, Phys. Rev. Lett. 90, 121302 (2003).

[92] R. Bousso, J. High Energy Phys. 0402, 025 (2004).

[93] R. Bousso, J. High Energy Phys. 0403, 054 (2004).

[94] R. Bousso, J. High Energy Phys. 0405, 050 (2004).

[95] W. G. Unruh, Phys. Rev. D14, 870 (1976).

[96] V. Frolov and I. Novikov, Phys. Rev. D48, 4545 (1993).

[97] A. O. Barvinsky, V. P. Frolov, and A. I. Zelnikov, Phys. Rev. D51, 1741 (1995).

[98] S. Carlip, Phys. Rev. D51, 632 (1995).

[99] C. Rovelli, Phys. Rev. Lett. 77, 3288 (1996).

[100] C. Teitelboim, Phys. Rev. D53, 2870 (1996).

[101] S. Carlip, Phys. Rev. D55, 878 (1997).

[102] C. Rovelli, Living Rev. Rel. 1, 1 (1998).

[103] C. Rovelli, gr-qc/9803024

[104] A. Ashtekar, J. Baez, A. Corichi and K. Krasnov, Phys. Rev. Lett. 80, 904 (1998).

[105] A. Ashtekar and K. Krasnov, gr-qc/9804039.

[106] S. Carlip, Phys. Rev. Lett. 82, 2828 (1999).

[107] S. Carlip, Phys. Rev. Lett. 88, 241301 (2002).

[108] G. 't Hooft, Nucl. Phys. B256, 727 (1985). 
[109] S. Mukohyama and W. Israel, Phys. Rev. D58, 104005 (1998).

[110] D. N. Page, Phys. Rev. D65, 024017 (2002).

[111] A. Sen, Mod. Phys. Lett. A10, 2081 (1995).

[112] A. Strominger and C. Vafa, Phys. Lett. B379, 99 (1996).

[113] C. G. Callan and J. M. Maldacena, Nucl. Phys. B472, 591 (1996).

[114] G. T. Horowitz and A. Strominger, Phys. Rev. Lett. 77, 2368 (1996).

[115] J. C. Breckenridge, R. C. Myers, A. W. Peet, and C. Vafa, Phys. Lett. B391, $93(199)$.

[116] C. V. Johnson, R. R. Khuri, and R. C. Myers, Phys. Lett. B378, 78 (1996).

[117] J. Maldacena and A. Strominger, Phys. Rev. Lett. 77, 428 (1996).

[118] J. C. Breckenridge, D. A. Lowe, R. C. Myers, A. W. Peet, A. Strominger, and C. Vafa, Phys. Lett. B381, 423 (1996).

[119] G. Horowitz, J. Maldacena, and A. Strominger, Phys. Lett. B383, 151 (1996).

[120] R. Dijkgraaf, E. Verlinde, and H. Verlinde, Nucl. Phys. B486, 77 (1997).

[121] G. T. Horowitz, D. A. Lowe, and J. M. Maldacena, Phys. Rev. Lett. 77430 (1996).

[122] J. M. Maldacena and L. Susskind, Nucl. Phys. B475, 679 (1996).

[123] I. R. Klebanov and A. A. Tseytlin, Nucl. Phys. B475, 164 (1996).

[124] E. Halyo, A. Rajaraman, and L. Susskind, Phys. Lett. bf B392, 319 (1997).

[125] M. Cvetic and A. A. Tseytlin, Nucl. Phys. B478, 181 (1996).

[126] J. M. Maldacena, Ph. D. Thesis, Princeton University (June 1996), hep-th/9607235.

[127] J. Maldacena and A. Strominger, Phys. Rev. D55, 861 (1997).

[128] G. T. Horowitz and D. Marolf, Phys. Rev. D55, 3654 (1997).

[129] J. Polchinski, "TASI Lectures on D-Branes," in C. Efthimiou and B. Greene, eds., Fields, Strings and Duality: TASI 96 (World Scientific, Singapore, 1997), p. 293; hep-th/9611050.

[130] G. T. Horowitz and J. Polchinski, Phys. Rev. D55, 6189 (1997).

[131] J. M. Maldacena and A. Strominger, Phys. Rev. D56, 4975 (1997). 
[132] C. Vafa, in E. Gava, A. Masiero, K.S. Narain, S. Randjbar-Daemi and Q. Shafi, eds., 1996 Summer School in High Energy Physics and Cosmology (World Scientific, Singapore, 1997), p. 66; hep-th/9702201.

[133] J. M. Maldacena, Nucl. Phys. Proc. Suppl. 61A, 111 (1998); Nucl. Phys. Proc. Suppl. 62, 428 (1998).

[134] T. Banks, W. Fischler, I. R. Klebanov, and L. Susskind, Phys. Rev. Lett. 80, 226 (1998).

[135] J. Maldacena, A. Strominger, E. Witten, J. High Energy Phys. 9712, 002 (1997).

[136] K. Sfetsos and K. Skenderis, Nucl. Phys. B517, 179 (1998).

[137] J. M. Maldacena, Adv. Theor. Math. Phys. 2, 231 (1998); Int. J. Theor. Phys. 38, 1113 (1999).

[138] A. Strominger, J. High Energy Phys. 9802, 009 (1998).

[139] A. W. Peet, Class. Quant. Grav. 15, 3291 (1998).

[140] D. Birmingham, I. Sachs, and S. Sen, Phys. Lett. B424 275 (1998).

[141] E. Witten, Adv. Theor. Math. Phys. 2, 505 (1998).

[142] A. Giveon, D. Kutasov and N. Seiberg, Adv. Theor. Math. Phys. 2, 733 (1998).

[143] A. Sen, "Developments in superstring theory," hep-ph/9810356.

[144] M. Cvetic, Fortsch. Phys. 48, 65 (2000).

[145] E. Witten, "New perspectives in the quest for unification," hep-ph/9812208.

[146] K. Skenderis, Lect. Notes Phys. 541, 325 (2000).

[147] S. S. Gubser, Ph.D. thesis, Princeton University (1998), hep-th/9908004.

[148] O. Aharony, S. S. Gubser, J. M. Maldacena, H. Ooguri and Y. Oz, Phys. Rept. 323, 183 (2000).

[149] D. Marolf, "String/M-branes for relativists," gr-qc/9908045.

[150] G. T. Horowitz, Class. Quant. Grav. 17, 1107 (2000).

[151] T. Damour, Annalen Phys. 11, 1 (2000) [Grav. Cosmol. Suppl. 6, 63 (2000 ANPYA,9,267-277.2000)].

[152] J. M. Maldacena, in J.A. Jaros and M.E. Peskin, eds. Proc. of the 19th Intl. Symp. on Photon and Lepton Interactions at High Energy LP99, Int. J. Mod. Phys. A15S1, 840 (2000) [eConf C990809, 840 (2000)].

[153] R. R. Khuri, Nucl. Phys. B 588, 253 (2000). 
[154] A. W. Peet, "TASI lectures on black holes in string theory," hep-th/0008241.

[155] S. R. Das and S. D. Mathur, Ann. Rev. Nucl. Part. Sci. 50, 153 (2000).

[156] R. C. Myers, Rev. Mex. Fis. 49S1, 14 (2003).

[157] R. R. Khuri, Nucl. Phys. B 617, 365 (2001).

[158] S. D. Mathur, "Where are the states of a black hole?," hep-th/0401115.

[159] L. Thorlacius, "Black holes and the holographic principle," hep-th/0404098.

[160] M. J. Duff, "Benchmarks on the brane," hep-th/0407175.

[161] L. Susskind, "Some speculations about black hole entropy in string theory," hep-th/9309145.

[162] W. K. Wootters and W. H. Zurek, Nature 299, 802 (1982).

[163] G. 't Hooft, Nucl. Phys. B 335, 138 (1990).

[164] G. 't Hooft, Phys. Scripta T36, 247 (1991).

[165] L. Susskind, L. Thorlacius, and J. Uglum, Phys. Rev. D48, 3743 (1993).

[166] L. Susskind, Phys. Rev. Lett. 71, 2367 (1993).

[167] L. Susskind and L. Thorlacius, Phys. Rev. D49, 966 (1994).

[168] L. Susskind, Phys. Rev. D49, 6606 (1994).

[169] D. A. Lowe, L. Susskind and J. Uglum, Phys. Lett. B327, 226 (1994).

[170] D. A. Lowe, J. Polchinski, L. Susskind, L. Thorlacius and J. Uglum, Phys. Rev. D52, 6997 (1995).

[171] L. Susskind and J. Uglum, Nucl. Phys. Proc. Suppl. 45BC, 115 (1996).

[172] D. V. Fursaev, Phys. Rev. D51, R5352 (1995).

[173] R. B. Mann and S. N. Solodukhin, Nucl. Phys. B 523, 293 (1998).

[174] R. K. Kaul and P. Majumdar, Phys. Rev. Lett. 84, 5255 (2000).

[175] S. Carlip, Class. Quant. Grav. 17, 4175 (2000).

[176] J. A. Cardy, Nucl. Phys. B 270, 186 (1986).

[177] H. W. J. Blöte, J. A. Cardy, and M. P. Nightingale, Phys. Rev. Lett. 56, 742 (1986).

[178] T. R. Govindarajan, R. K. Kaul, and V. Suneeta, Class. Quant. Grav. 18, 2877 (2001). 
[179] M. Bañados, C. Teitelboim, and J. Zanelli, Phys. Rev. Lett. 69, 1849 (1992).

[180] M. Bañados, M. Henneaux, C. Teitelboim, and J. Zanelli, Phys. Rev. D48, 1506 (1993).

[181] S. K. Rama, Phys. Lett. B566 152 (2003).

[182] G. Gour, Phys. Rev. D66, 104022 (2002).

[183] M. Domagala and J. Lewandowski, Class. Quant. Grav. 21, 5233 (2004).

[184] K. A. Meissner, Class. Quant. Grav. 21, 5245 (2004).

[185] A. Ghosh and P. Mitra, "On the log correction to the black hole area law," gr-qc/0401070.

[186] A. Ghosh and P. Mitra, "An improved estimate of black hole entropy in the quantum geometry approach," gr-qc/0411035.

[187] A. Ghosh and P. Mitra, Phys. Rev. Lett. 73, 2521 (1994).

[188] S. Das, R. K. Kaul, and P. Majumdar, Phys. Rev. D63, 044019 (2001).

[189] S. Das, P. Majumdar, and R. K. Bhaduri, Class. Quant. Grav. 19, 2355 (2002).

[190] S. Mukherji and S. S. Pal, JHEP 0205, 026 (2002).

[191] S. Nojiri, S. D. Odintsov, and S. Ogushi, Int. J. Mod. Phys. A18 3395 (2003).

[192] C. Castro and A. Granik, Found. Phys. 33, 445 (2003).

[193] A. Chatterjee and P. Majumdar, "Black hole entropy: quantum vs. thermal fluctuations," gr-qc/0303030.

[194] M. R. Setare, Phys. Lett. B573, 173 (2003).

[195] M. R. Setare, Eur. Phys. J. C33, 555 (2004).

[196] G. Amelino-Camelia, M. Arzano, and A. Procaccini, Phys. Rev. D70, 107501 (2004).

[197] S. Hod, Class. Quant. Grav. 21, L97 (2004).

[198] Y. S. Myung, Phys. Lett. B579 205 (2004).

[199] M. I. Park, "Testing holographic principle from logarithmic and higher order corrections to black hole entropy," hep-th/0402173.

[200] A. Ashtekar and J. Lewandowski, Class. Quant. Grav. 21, R53 (2004).

[201] A. J. M. Medved, "A comment on black hole entropy or does Nature abhor a logarithm?" gr-qc/0406044 
[202] A. Chatterjee and P. Majumdar, "Mass and charge fluctuations and black hole entropy," gr-qc/0409097.

[203] S. S. More, "Higher order corrections to black hole entropy," gr-qc/0410071.

[204] A. J. M. Medved, "A follow-up to 'Does Nature abhor a logarithm?' (and apparently she doesn't)," gr-qc/0411065.

[205] I. B. Khriplovich, "Holographic bound and spectrum of quantized black hole," gr-qc/0411109.

[206] S. W. Hawking and D. N. Page, . Math. Phys. 87, 577 (1983).

[207] D. N. Page, Gen. Rel. Grav. 13, 1117 (1981).

[208] V. P. Frolov, Int. J. Mod. Phys. D 5, 649 (1996).

[209] R. M. Wald, in R. M. Wald, ed., Black Holes and Relativistic Stars (University of Chicago Press, Chicago, 1998); gr-qc/9702022.

[210] T. Jacobson, "Black hole thermodynamics today," gr-qc/9801015.

[211] V. P. Frolov and D. V. Fursaev, Class. Quant. Grav. 15, 2041 (1998).

[212] S. Mukohyama, Ph.D. thesis, Kyoto University (1998); gr-qc/9812079.

[213] C. Kiefer, in P. Fre, V. Gorini, G. Magli and U. Moschella, eds., Classical and Quantum Black Holes (Institute of Physics Publishing, Bristol, 1999), p. 19.

[214] T. Jacobson, in C. P. Burgess and R. C. Myers, eds., General Relativity and Relativistic Astrophysics: Proceedings of 8th Canadian Conference on General Relativity and Relativistic Astrophysics, Montreal, Quebec, Canada, 10-12 June 1999 (American Institute of Physics, Melville, N.Y., 1999); gr-qc/9908031.

[215] S. Mukohyama, "Aspects of black hole entropy," gr-qc/9912103.

[216] R. M. Wald, Living Rev. Rel. 4, 6 (2001).

[217] P. Majumdar, Pramana 55, 511 (2000).

[218] P. Majumdar, "Black hole entropy: Classical and quantum aspects," hep-th/0110198.

[219] C. Kiefer, "Quantum aspects of black holes," astro-ph/0202032.

[220] W. Israel, Lect. Notes Phys. 617, 15 (2003).

[221] T. Jacobson, "Introduction to quantum fields in curved space-time and the Hawking effect," gr-qc/0308048.

[222] T. Damour, "The entropy of black holes: A primer," hep-th/0401160.

[223] S. Das, Pramana 63, 797 (2004).

[224] D. V. Fursaev, "Can one understand black hole entropy without knowing much about quantum gravity?" gr-qc/0404038. 